\title{
Refined sea state analysis from an array of four identical directional buoys deployed off the Northern Cornish coast (UK)
}

\author{
Jean-Baptiste Saulnier $^{\mathrm{a}, *}{ }^{\text {, Christophe Maisondieu }}{ }^{\mathrm{b}}$, Ian Ashton ${ }^{\mathrm{a}}$, George H. Smith ${ }^{\mathrm{a}}$
}

\author{
${ }^{a}$ Renewable Energy Group, C.E.M.P.S., University of Exeter, Cornwall Campus, PENRYN, Cornwall TR10 9EZ, \\ United Kingdom

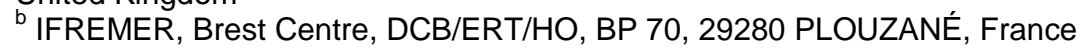 \\ *: Corresponding author : Jean-Baptiste Saulnier, email address : j.saulnier@exeter.ac.uk
}

\begin{abstract}
:
This paper presents an analysis of sea states carried out from extended wave measurements realized near the Wave Hub wave energy test facility in Cornwall (UK). The space directional information is derived from the spectro-directional processing of time-domain data provided by an array of four independent SEAWATCH Mini II displacement buoys separated by an approximate average distance of $500 \mathrm{~m}$. It is observed that, even though the size of the array is small compared to the local wave trains' length, the estimation of the directional spectra - using maximum entropy and likelihood methods - may sometimes exhibit certain space variability over the array. It was also observed that the tidal currents variations produced a significant influence upon the wave directional estimation and wave spectral parameters. Spectro-directional partitioning and wave system tracking were subsequently applied and this emphasized the influence of tide variations upon the individual swells and wind-seas as measured by the buoys. This paper, therefore, also illustrates the relevance of applying partitioning and tracking procedures for the identification of wave systems in oceanographic and wave energy studies.
\end{abstract}

\section{Highlights}

Wave directional spectra are estimated by an array of four identical wave buoys. The variability of wind-sea spectral estimates is consistent with random error. Low-frequency swell systems as measured by the array are subject to uncertainty. An analysis of the tide's influence on wave characteristics and systems is made.

Keywords: Directional wave spectrum ; Directional buoys ; Buoys array ; Maximum entropy method ; Maximum Likelihood Method ; Sea state ; Wave system ; Spectro-directional partitioning; Wave stationarity/homogeneity ; Wave/tide interactions 


\section{Introduction}

Wave energy converters (WECs), although still under development, have now reached the stage where they require testing and proving at full stage and in real seas. Though indispensable, tank testing, because of the limited scale and limited wave randomness that can be reproduced in a small and/or shallow depth tank (without tide, currents, wind effects, etc.), is not sufficient to provide a robust, reliable final assessment of either the efficiency or survivability of WECs. In view of the importance of obtaining full scale, 'real' sea state conditions, the recent years have seen the development of several maritime sites for deployment and testing of technologies at intermediate or full scale, with or without seabedfixed grid connection. In Europe in particular, the European Marine Energy Centre (EMEC) test site in the Orkney Islands (Scotland) has seen several deployments of the full-scale Pelamis device (2004-2007). The quarter-scale Wave Dragon (2003) and tenth-scale Wave Star (2006) prototypes were deployed in the Danish sheltered area of Nissum Bredning. In Ireland, the Ocean Energy test site located within the Galway Bay (Marine Institute \& Sustainable Energy Ireland) was chosen to moor the quarter-scale Wave Bob (2006) and half-scale OE Buoy (2007) prototypes. In Sweden, linear generators are currently tested on the Lisekil test zone (Uppsala University). In France, the construction of the SEM-REV (Site d'Expérimentation en Mer pour la Récupération de l'Energie des Vagues) near the town of Le Croisic is being finalized (Ecole Centrale de Nantes). The Iberian Peninsula is also committed to this research field with the BIMEP (Blscay Marine Energy Platform) in Spain and the Portuguese pilot zone in São Pedro de Moel, which were both announced in 2008 and are expected to be operational within the next few years.

The offshore 20MW grid-connected Wave Hub test facility in Cornwall is one of these promising projects. First announced in 2003, it is now operational for WEC experimentation and energy transport to the coast, with the final deployment of the undersea cable to a 'hub' in some $50 \mathrm{~m}$ water depth. Along with the development of the Wave Hub site for commercial deployment of small arrays, an extensive research and measurement campaign has been undertaken by PRIMaRE (a joint initiative between the universities of Exeter and Plymouth). For the purposes of wave and current measurement, an array of instruments - four directional buoys - was installed some $4 \mathrm{~km}$ South-West from the hub. The buoys deliver synchronized time-series at $2 \mathrm{~Hz}$.

In this study, a cross-spectral analysis is applied to the recorded motion signals to estimate either the spectral density and the four first directional Fourier coefficients, or more simply the cross-spectral matrix. Maximum Entropy (MEM) and Maximum Likelihood (MLM) methods are implemented to estimate the directional distribution $D(f, \theta)$ of the directional wave spectrum $S(f, \theta)$. The directionality of waves is therefore compared from several independent synchronized measurements so that the wave homogeneity (i.e. the spatial stationarity) assumption can be examined over the considered area. This paper particularly focuses on the measurements realised in the month of February 2010. Each sea state spectrum is estimated using the methods referred to above, and two different characterisations are proposed, based on the recent paper of Saulnier et al. [1]. A sea state is considered either as (i) close to unimodal and characterised by a set of overall parameters (energy, period, direction, bandwidth and directional spreading), or (ii) as a multimodal process - as it often is in nature. The second hypothesis then requires that the identification and individual characterisation of each wave system making up the sea state (swells and wind-seas) be undertaken. In this paper, specific partitioning and wave system tracking algorithms are employed to separate the individual characteristics of either system. Local wind and tidal information are also included in the study so that their possible influence on the sea states and the wave systems can be emphasized. This directional analysis permits an enhanced understanding of the physical evolution of the sea states. 
The paper is outlined as follows. Section 2 presents the characteristics of the measurement buoy array and environmental data. Section 3 details the cross-spectral analysis and the directional estimation methods used in this study. Section 4 reviews the two different sea state characterisations adopted in the study, which involve overall parameters and a specific parameterisation applied to each individual wave system. Section 5 presents and discusses some results of the directional spectra derived from the four synchronized buoys as well as the time evolution of overall and system parameters. Section 6 draws some conclusions and further proposed investigations arising from this work.

\section{The Wave Hub test facility and the measurement buoy array}

The Wave Hub test facility is located $15 \mathrm{~km}$ off the North coast of Cornwall (South West England, see Fig. 1). The vision was to provide an electrical infrastructure for connection into the UK grid systems, for small arrays of WECs. The power cable and connection hub has been designed to connect up to four, different, 4-5MW rated WECs to the local grid through a $25 \mathrm{~km}, 33 \mathrm{kV}$ subsea cable (currently operating at $11 \mathrm{kV}$ ). This was installed on the seabed in early September 2010.

The wave measurement array was deployed to investigate particular aspects of spatial and statistical variability in the observed sea states. For logistical and survival reasons the array has been initially located about $4 \mathrm{~km}$ from the hub. The array, in 2010 , consisted of four SEAWATCH Mini II directional buoys provided by the Norwegian company Fugro OCEANOR (http://www.oceanor.no/, last visited on 14/09/2011). Each buoy houses accelerometers and compass and records its own displacements in the orthogonal coordinate reference system (Heave $z$ /East $x$ /North $y$, on the geographic compass). The array as configured from January to April 2010 is depicted in Figure 2. The local mean water depth over the array is about $40 \mathrm{~m}$. Typical features of the local tidal flows are $1 \mathrm{~m} / \mathrm{s}$ speed coming from West-South-West $\left(230^{\circ}\right)$ with amplitude levels varying from $1 \mathrm{~m}$ to $3 \mathrm{~m}$.

The individual buoys are, in this paper, differentiated by a capital letter ('A' to ' $D$ ') standing for the space location. Each time-series consists of 2048 points recorded at $2 \mathrm{~Hz}$ sampling rate, giving a record time of some 17 minutes with records being stored every 30 minutes on the hour and half-hour (48 motions datasets per day). All four buoys are synchronized together according to GMT from the satellite GPS, so that they record data in time synchronisation. Table 1 gives some technical details of the array (positions, depth...) for the month of February 2010, which is the focus for this paper. North reference onboard is Magnetic North, which in turn defines the East direction (see §3.1). The average magnetic declination in North Cornwall for the period January to April 2010 is estimated about $3.6^{\circ}$ towards West. The data were quality checked according to the methods described in [2], and it should be noted that the level of failure rate for the considered month of measurements is extremely low $(<0.1 \%)$.

To make this study more complete, wind and tidal data related to the month of February 2010 were also collected. Wind data $\left(10 \mathrm{~m}\right.$-height mean speed $U_{\mathrm{w}}$ and origin direction $\left.\theta_{\mathrm{w}}\right)$ were provided by the British MetOffice from the North Atlantic and European $12 \mathrm{~km}$ configuration of an atmospheric unified model. The model output grid point is $50^{\circ} 18^{\prime} 00^{\prime \prime} \mathrm{N}$, $05^{\circ} 36^{\prime} 36^{\prime \prime} \mathrm{W}$, which is located some $4 \mathrm{~km}$ South-East from the centre of the buoy array (see Fig. 1). Hourly results are produced by the model only so that half-hour data are estimated using cubic spline interpolation. Tidal data were calculated using the POLPRED ${ }^{\odot}$ offshore tidal computation software version 2.4.1.1 (Proudman Oceanographic Laboratory, UK) which outputs the level, depth-integrated flow speed and direction of the tide every minute at the location $50^{\circ} 18^{\prime} 30^{\prime \prime} \mathrm{N}, 05^{\circ} 40^{\prime} 05^{\prime \prime} \mathrm{W}$, central to the buoy array. 


\section{Spectral processing and directional estimation methods}

\subsection{Cross-spectral analysis}

In offshore engineering, a sea state is commonly represented by its directional spectral density $S(f, \theta)$ which describes the distribution of wave energy over frequencies and directions. It is usually decomposed into [3]

$$
S(f, \theta)=S(f) \cdot D(f, \theta)
$$

where $S(f)$ is the (omnidirectional) variance spectral density of the water elevation and the spectro-angular function $D(f, \theta)$ is denoted by the term directional distribution function. This is assumed to be positive and must satisfy the following condition of normalisation

$$
\int_{0}^{2 \pi} D(f, \theta) d \theta=1
$$

From in situ measurements, the estimation of $S(f)$ requires water elevation data only whereas $S(f, \theta)$ in addition requires the measurement of at least two more wave quantities (or at different positions). For the case of Heave/Sway/Surge buoys, the device records time-series related to water elevation $(z)$, East $(x)$ and North $(y)$ particle displacements respectively. The magnetic declination $\delta_{\mathrm{md}}$ (positive towards West) due to the North reference used by the buoy must be taken into consideration, even though the declination value generally is small (less than $4^{\circ}$ towards West in North Cornwall in February 2010 as said previously). The new $x$ and $y$ signals against geographical North and related East directions respectively are calculated as

$$
\begin{aligned}
& \tilde{x}=x \cos \delta_{m d}-y \sin \delta_{m d} \\
& \tilde{y}=y \cos \delta_{m d}+x \sin \delta_{m d}
\end{aligned}
$$

where $\delta_{m d}$ is expressed in radians.

The estimation of the directional spectrum requires the computation of the cross-spectra $G_{\mathrm{mn}}(f), 1 \leq m, n \leq 3$, of the motion signals. Assuming wave linear potential theory, indeed, these are related to $D(f, \theta)$ by (see e.g. $[4,5]$ )

$$
G_{m n}(f)=S(f) \cdot \int_{0}^{2 \pi} g_{m}(f, \theta) \overline{g_{n}(f, \theta)} \cdot D(f, \theta) d \theta
$$

where $g_{\mathrm{m}}(f, \theta)=H_{\mathrm{m}}(f, \theta)^{*} \exp \left[\mathbf{k} \cdot \mathbf{x} \cdot \mathbf{x}_{\mathrm{m}}\right]=H_{\mathrm{m}}(f, \theta)$ stand for the (space) transfer functions linking signal $m$ to water elevation (all measured at the same physical point $\mathbf{x}=0$ here since a directional buoy is a single-point measurement device) with $\mathbf{k}$ the wave number vector defined in the waterplane. For a Heave $(1, z)$-East $(2, x)$-North $(3, y)$ displacement buoy, these are given by

$$
\begin{aligned}
& g_{1}(f, \theta) \equiv H_{1}(f, \theta)=1 \\
& g_{2}(f, \theta) \equiv H_{2}(f, \theta)=i \cos (\theta) / \tanh (k d) \\
& g_{3}(f, \theta) \equiv H_{3}(f, \theta)=i \sin (\theta) / \tanh (k d)
\end{aligned}
$$


where the wave-number modulus $k$ is calculated from the dispersion equation: $\omega^{2}=4 \pi^{2} f^{2}=$ $g k \tanh (k d)$ at depth $d$. It is easily shown that quantities $G_{m n}(f)$ and $G_{n m}(f)$ are complex conjugate, so that only six cross-spectra need to be estimated in reality. Let us denote by $\mathbf{G}=\left[G_{\mathrm{mn}}(f)\right]$ the corresponding $6 \times 6$ cross-spectral matrix for a given frequency and by $C_{\mathrm{mn}}(f)$ the real (co-spectrum) part of the cross-spectra. Thus, three auto-spectra $\left(\hat{C}_{11}(f), \hat{C}_{22}(f)\right.$, $\left.\hat{C}_{33}(f) \in \mathrm{R}\right)$ and three cross-spectra $\left(\hat{G}_{12}(f), \hat{G}_{13}(f), \hat{G}_{23}(f) \in \mathrm{C}\right)$ have to be computed at each frequency. Using classical cross-spectral analysis, the resulting estimates are frequently contaminated by errors inherent to both the measuring apparatus and the estimation technique (see e.g. $[6,7,8,9]$ ). Practically, running a periodogram technique (Fast Fourier Transform, FFT) with tapering and possibly segment overlapping (see Welch [10]) should yield quite a reliable estimation $\hat{G}_{\mathrm{mn}}(f)$ as long as the records' quality is ensured, so that the equality $\mathbf{G} \equiv \hat{\mathbf{G}}$ may be reasonably further on assumed. Here, a 256-point cross-spectral analysis is carried out with $50 \%$ segment overlapping and Hanning-window edge tapering. According to Welch's methodology, this results in cross-spectral estimates with about 28 equivalent degrees of freedom (DOFs) for 2048-point time-series.

Many methods have been proposed to estimate $D(f, \theta)$ in a consistent way from the little information available. Some of these methods are presented hereafter. A comprehensive and thorough review of the available directional estimation methods can be found in [5].

\subsection{Fourier series decomposition}

The directional distribution may be seen as a positive $2 \pi$-periodic trigonometric integrable function. As such, it can be decomposed into the Fourier series

$$
\begin{aligned}
D(f, \theta) & =\frac{1}{2 \pi} \cdot \sum_{n=-\infty}^{+\infty} c_{n}(f) \cdot e^{i n \theta} \\
& =\frac{a_{0}(f)}{2 \pi}+\frac{1}{\pi} \cdot \sum_{n=1}^{+\infty} a_{n}(f) \cdot \cos (n \theta)+b_{n}(f) \cdot \sin (n \theta)
\end{aligned}
$$

where

$$
\begin{aligned}
& c_{n}(f)=\int_{0}^{2 \pi} D(f, \theta) \cdot e^{-i n \theta} d \theta, \quad c_{-n}(f)=\overline{c_{n}(f)} \\
& a_{n}(f)=\int_{0}^{2 \pi} D(f, \theta) \cdot \cos (n \theta) d \theta=\operatorname{Re}_{e}\left\{c_{n}(f)\right\} \\
& b_{n}(f)=\int_{0}^{2 \pi} D(f, \theta) \cdot \sin (n \theta) d \theta=-\operatorname{Im}\left\{c_{n}(f)\right\}
\end{aligned}
$$

Thus $a_{0}(f)=1$. If one could accurately estimate the Fourier coefficients up to quite a high order, the estimation of $D(f, \theta)$ would certainly be quite acceptable. It is possible to provide such high-order coefficients in special circumstances - for example through the use of large gauge arrays (e.g. [11]). In practice though, it is rare to compute orders higher than 2, which is the case for directional buoys. The $2^{\text {nd }}$-order truncated Fourier series estimator has been shown to be unreliable, indeed, for it usually produces negative values. To remedy this issue, a weighted Fourier series was proposed by Longuet-Higgins et al. [3], which yet exaggeratedly broadens the peaks and is, for this reason, not used in general. Another 
approach to estimate the directional distribution function is to fit analytic pre-defined shapes. Such parametric estimators are most often chosen as unimodal (i.e., one peak only) and symmetrical about the mean wave direction, introducing thus a strong assumption on the directional distribution, which might not always be true in reality. The most popular shape is the $\cos ^{2 s}$ function [3], which is characterised by a frequency-dependent mean direction $\theta_{0}(f)$ and directional spreading factor $s(f)$. Both can be estimated from the $1^{\text {st }}$-order Fourier coefficients (Eq. (7-9)) as

$\theta_{0}(f) \equiv \hat{\theta}_{1}(f)=-\arg \left[\hat{c}_{1}(f)\right]$

and

$$
s(f) \equiv \hat{s}_{1}(f)=\frac{\left|\hat{c}_{1}(f)\right|}{1-\left|\hat{c}_{1}(f)\right|}
$$

Alternative expressions related to the $2^{\text {nd }}$-order Fourier coefficients do exist and can be found in e.g. [5]. Obviously, the directional estimator is consistent with one pair ( $1^{\text {st }}-$ or $2^{\text {nd }}$-order$)$ of Fourier coefficients only. The estimation quality of these coefficients is discussed later in Section 5. More parametric functions can be found in [12]. Application of bimodal parametric shapes (i.e., exhibiting two peaks) may be found in e.g. [13,14]. However, these methods are not used in this work.

\subsection{Maximum Likelihood Methods (EMLM/IMLM)}

More sophisticated methods have been introduced in ocean engineering analyses, particularly to account for the possible bimodality of directional seas from the little estimated information available. As simply and soundly summarized by Arribas and Egozcue [8], these estimators can be seen "as an interpolation of $D(f, \theta)$ by a rational trigonometric function whose Fourier coefficients are known". One of these methods is the (Extended) Maximum Likelihood Method ((E)MLM). Its general use to any in situ wave measuring set-up was described by Isobe et al. [4] but the original theory is due to Capon [15], who applied it first in seismic processing. The final ML estimator may be expressed as

$$
\hat{D}_{E M L M}(f, \theta)=\frac{\kappa}{{ }^{H} \mathbf{g}(f, \theta) \cdot \hat{\mathbf{G}}^{-1}(f) \cdot \mathbf{g}(f, \theta)}
$$

where ${ }^{H}$ left-hand superscript denotes Hermitian transpose, $K$ is a constant ensuring the normalisation of the directional estimator and $\mathbf{g}$ denotes the space transfer function vector (Eq. (4)). In practice - especially when $\hat{\mathbf{G}}(f)$ is not positive semi-definite - the inversion of the cross-spectral matrix may be tricky and a generalized inversion (using e.g. pinv() function in Matlab) is recommended [16]. The EMLM estimator is therefore straightforward to compute. However, it is commonly commented that the estimated distribution is not consistent with the original cross-spectra. Furthermore, the EMLM is considered to induce energy diffusion with the resulting distribution function often looking 'smeared' (H.E. Krogstad, [16]). Several iterative schemes (termed the IMLM) have been proposed to rectify these drawbacks. In [16], the following scheme is applied

$$
\begin{aligned}
& { }^{n+1} \hat{D}_{I M L M}={ }^{n} \hat{D}_{I M L M}+\omega_{R} \cdot\left[\hat{D}_{E M L M}-M\left({ }^{n} \hat{D}_{I M L M}\right)\right] \\
& { }^{0} \hat{D}_{I M L M}=\hat{D}_{E M L M}
\end{aligned}
$$


where $\omega_{\mathrm{R}}$ is a relaxation parameter (taken equal to 0.3 here) and left-hand superscripts denote the rank of iteration. The application $M: D \rightarrow D_{\text {EMLM }}$ calculates the EMLM estimate from the cross-spectral matrix (or the Fourier coefficients) of a given directional distribution function $D$ (Eq. (4)). The new IMLM estimate is normalized after each loop and the scheme stops when a given convergence criterion is met (an alternative IMLM scheme can be found in e.g. [17]). In the present study, about 25 iterations were necessary to obtain less than a $0.1 \%$ variation on the spectro-angular peak value. The IMLM distribution looks in general less broad compared to the EMLM and the important energy diffusion is much reduced.

The IMLM perfectly suits multi-component measurement arrays since it does not necessarily require the explicit knowledge of the Fourier coefficients (see e.g. [9,16,18,19]). For directional buoy data, both EMLM and IMLM are easy to implement but many authors $[5,18,20]$ argued that maximum entropy methods (MEMs, see $\$ 3.4)$ are much preferable in this case. Some refinements of this method may be found in e.g. Haug and Krogstad [21] (combined MLM/MEM), Arribas and Egozcue [8] (normalized MLM estimators), and Marsden and Juzko [22] (eigenvector method).

\subsection{Maximum Entropy Methods}

Maximum Entropy (ME) Methods applied to directional buoy data have been widely implemented by oceanographers from the 80 s onwards $[18,20,23]$. Two entropy definitions are usually adopted: on the one hand, John P. Burg's formulation (called here Burg's MEM and denoted by BMEM) as [24]

$$
H_{B M E M}(D)=-\int_{0}^{2 \pi} \ln (D(\theta)) d \theta
$$

and, on the other hand, Claude E. Shannon's (called here Shannon's MEM, or Maximum Entropy Principle, MEP) as

$$
H_{M E P}(D)=-\int_{0}^{2 \pi} D(\theta) \ln (D(\theta)) d \theta
$$

both defined for a given frequency $f$. MEMs were originally used in probability theory and are applied to directional wave data by considering the directional distribution function $D(\theta)$ as a probability density function. The estimation of $D(\theta)$ is therefore based on the maximization of one of either Burg's or Shannon's entropy functional under some constraints related to the field measurements: in the case of directional buoys, the directional estimator has to match the estimated Fourier coefficients up to the $2^{\text {nd }}$-order.

It is shown [24] that the general solution of the Burg ME problem based on the entropy integral in Equation (14) takes the form

$$
\hat{D}_{B M E M}(f, \theta)=\frac{1}{2 \pi} \cdot \frac{\sigma_{e}^{2}(f)}{\left|1-\varphi_{1}(f) e^{-i \theta}-\ldots-\varphi_{n}(f) e^{-n i \theta}\right|^{2}}
$$


where parameters $\varphi_{1}(f) \ldots \varphi_{\mathrm{n}}(f)$ are the solution of the Yule-Walker equation (see [20]) and $\sigma_{e}^{2}(f)=1-\varphi_{1}(f) \overline{\hat{c}}_{1}(f)-\cdots-\varphi_{n}(f) \overline{\hat{c}}_{n}(f)$. For the particular case of directional buoys, this results in

$$
\begin{aligned}
& \varphi_{1}=\frac{\hat{c}_{1}-\hat{c}_{2} \overline{\hat{c}}_{1}}{1-\left|\hat{c}_{1}\right|^{2}} \\
& \varphi_{2}=\hat{c}_{2}-\hat{c}_{1} \varphi_{1} \\
& \sigma_{e}^{2}=1-\overline{\hat{c}}_{1} \varphi_{1}-\overline{\hat{c}_{2}} \varphi_{2}
\end{aligned}
$$

which is straightforward to compute and implement (frequency dependence has been omitted for convenience).

As far as Shannon's entropy is concerned, regarding directional buoy data, it is shown [23] that the directional estimator solving the ME equation system may be expressed as

$$
\hat{D}_{M E P}(f, \theta)=\exp \left[\lambda_{0}(f)+\lambda_{1}(f) \cos (\theta)+\lambda_{2}(f) \sin (\theta)+\lambda_{3}(f) \cos (2 \theta)+\lambda_{4}(f) \sin (2 \theta)\right]
$$

where $\left(\lambda_{i}\right)_{0 \leq i \leq 4}$ are frequency-dependent Lagrange multipliers consistent with the estimated Fourier coefficients. The computation of the MEP estimator therefore is somewhat more computationally time-consuming than BMEM. This method is reviewed here for completeness but shall not be illustrated in the following, for very similar directional estimations to BMEM - after spectro-angular smoothing (see §3.5) - were found.

Several authors (e.g. $[13,20,25])$ have drawn up comparative studies between both methods based on in situ measurements, especially buoys. They all comment that Burg's ME estimator sometimes produces artificial double-peaked shapes in known unimodal sea states. In addition, the peaks generally are found to be narrower than the target directional shape. These drawbacks are however compensated for by a remarkable peak resolution that can be achieved using this method. In the present data set, it was observed that the occurrences of double peaks were very limited provided that the precision level of the crossspectral estimates was sufficiently high.

Recently however, Saulnier et al. [1] have demonstrated from an analysis of WAVEC buoy data recorded in Portugal that MEMs could sometimes produce another type of directional artefact. This is observed as a spurious secondary peak with an angular separation of approximately $180^{\circ}$ from the main peak. It is thought that such methods may be sensitive to the signals' noise level in buoy recordings (distinct from the sampling error, see [6]), especially in the estimation of low-frequency wave components.

\subsection{Spectro-angular smoothing}

Estimated directional spectra, indeed, are often altered by noise, whether it is induced by measurement (instruments, sampling variability...) or by the numerical estimation method (spectral leakage, aliasing...). In order to apply the partitioning method described later in $\S 4.2$ which allows the identification of wave systems inherent within the sea-states, it is necessary to filter this noise somehow. This is mostly necessary to avoid the consideration of systems which possibly are artefacts. The algorithm adopted here consists in averaging the spectroangular matrix $\hat{S}\left(f_{\mathrm{i}}, \theta_{\mathrm{j}}\right)$ by convolution with a $2 \mathrm{D} 3 \times 3$ cell rectangular window, as described in 
[26]. Here, the smoothing is possibly repeated more than once. The quantity of smoothing is determined from visual observation of the directional spectrum estimates during the wave system identification (see §4.2): the criterion is based on the assumption that the number of peaks identified in the first step of the spectral-directional partitioning procedure must ideally be less than 10 . With the present data $\left(\hat{S}\left(f_{i}, \theta_{\mathrm{j}}\right)\right.$ is a 64 by 24 matrix, with $\Delta f=1 / 128=$ $7.8125 .10^{-3} \mathrm{~Hz}$ and $\left.\Delta \theta=15^{\circ}\right)$, it was observed that one or two loops of smoothing are sufficient (see Section 5).

In the following, the applied directional estimation methods are denoted by BMEM (Burg's MEM) and IMLM plus a final number indicating the spectral smoothing level. For instance, 'BMEM2' means re-composition using Burg's MEM followed by two smoothing loops.

\section{Sea state and wave system characterisation}

\subsection{Overall parameters}

A given sea state may be characterised by a set of overall parameters, which are directly calculated from the directional spectrum. The spectral and directional parameterisation adopted here follows that presented in [1]. Though the application of the following expressions involves spectro-angular estimates, hats are omitted for convenience.

The $\left(n^{\text {th }}\right.$-order) moments of the variance spectral density are calculated as

$$
m_{n}=\int f^{n} S(f) d f=\iint f^{n} S(f, \theta) d f d \theta
$$

The (spectral) significant wave height is then computed as

$$
H_{m 0}=4 \sqrt{m_{0}}
$$

Peak period and peak direction, defined as

$$
\left(f_{p}, \theta_{p}\right)=\left\{(f, \theta) \mid S\left(f_{p}, \theta_{p}\right)=\max [S]\right\}
$$

are used here to localise the dominant peak in each sea state, which in turn permits the raw tracking of the main wave systems in time if one looks at a continuous sequence of directional spectra. The mean overall direction and directional spreading are respectively computed as

$$
\theta_{m}=\arg \left[\int S(f) e^{i \theta_{1}(f)} d f\right] \equiv \tan ^{-1}\left(\frac{\int S(f) \sin \left(\theta_{1}(f)\right) d f}{\int S(f) \cos \left(\theta_{1}(f)\right) d f}\right)
$$

where $\theta_{1}(f)$ (radians) is estimated according to Equation (10), and

$$
\sigma_{m}=\arg \left[\int S(f) e^{i \sigma_{1}(f)} d f\right] \equiv \tan ^{-1}\left(\frac{\int S(f) \sin \left(\sigma_{1}(f)\right) d f}{\int S(f) \cos \left(\sigma_{1}(f)\right) d f}\right)
$$

where the $1^{\text {st }}$-order spreading coefficient $\sigma_{1}(f)$ (radians) is related to the directional Fourier coefficients by (see [6]) 


$$
\sigma_{1}(f)=\sqrt{2\left[1-\mid c_{1}(f)\right]}=\sqrt{2\left[1-\sqrt{a_{1}^{2}(f)+b_{1}^{2}(f)}\right]}
$$

These directional parameters can be calculated either directly from the estimated coefficients or, alternatively, from those re-computed from the final estimation of the directional spectrum (using Eq. (1) \& (7-9)). In the latter case, their value may vary according to the directional estimation method and the spectral smoothing procedure. This option is adopted in the following for the estimation of $\theta_{\mathrm{m}}$ and $\sigma_{\mathrm{m}}$. An overall spectral bandwidth figure (in $\mathrm{Hz}$ ) is also calculated, as [27]

$$
\Lambda=\frac{m_{0}^{2}}{\int S^{2}(f) d f}
$$

This bandwidth parameter is not the conventional one, quoted in most ocean engineering texts (i.e. either $\varepsilon=\left(1-m_{2}{ }^{2} /\left(m_{0} m_{4}\right)\right)^{1 / 2}$ or $\left.v=\left(\left(m_{0} m_{2}\right) / m_{1}{ }^{2}-1\right)^{1 / 2}\right)$. However, parameter $\Lambda$ has been shown [28] to yield an explicit and stable measure of the spectral bandwidth, in particular on what regards the sensitivity to cut-off high frequency. It is important to remind that, in our context, the estimation of parameters $H_{\mathrm{m} 0}, \Lambda$ and, to a certain extent, $f_{\mathrm{p}}$ (Eq. (21)), should be hardly affected by the directional estimation method (non-directional parameters).

\subsection{Wave system characterisation}

The general unimodal representation of the sea-states does not allow for an accurate description of their physical reality, with sea states often consisting of a combination of two or more wave systems. For example, Guedes Soares [29] quotes $22 \%$ of measured sea states as being bimodal on average in the Atlantic Ocean. More recently, Kerbiriou et al. [30] demonstrated that the occurrence of such sea states was about $40 \%$ in their wave climate study based on global wave hindcast data for the Bay of Biscay in 2004-2005. In order to provide a realistic insight of the sea state represented by this dataset, a partitioning and system tracking algorithm [30] was applied to histories of estimated directional spectra with the goal to identify constituent wave systems, namely wind-sea(s) and swell(s). The basis for the discussion below can be found in greater detail in the landmark work of Kerbiriou et al., which is also addressed in [1]. The partitioning process is realised by a watershed routine (isolation of catchment areas based on the steepest ascent path technique, see e.g. [31]) available in the MATLAB ${ }^{\circledR}$ Image Processing Toolbox, which allows for peaks identification in the frequency-direction plane. A low-energy system which could be considered as noise is merged into the closest significant neighbouring system if it represents less than $4 \%$ of the variance $\left(m_{0}\right)$ of the latter, and if the peak frequencies and directions are separated by less than 2 frequency bins and $40^{\circ}$ respectively. Wave systems are then classified as swell or wind-sea according to a separation frequency criterion evaluated from wind speed, which is here estimated as (see e.g. [32])

$$
f_{s}=\frac{g}{2 \pi \beta U_{w}}
$$

where $\beta$ is a parameter generally taken between 1 and $1.5(\beta=4 / 3$ here). This formulation derives from the local wave age criterion, which states that the phase celerity of waves in deep water cannot exceed a certain fraction $(\beta)$ of the local wind speed $U_{w}$ (see e.g. [33]). Thus, wind-seas should normally exhibit peak frequencies higher than $f_{\mathrm{s}}$, especially during wave growth phases. Then, analytical functions based on the JONSWAP formulation [34] for the frequency distribution and on $\cos ^{2 s}$ for the directional distribution [3] are fitted to each system so that the latter can be described using the usual parametric representation based on a set of five parameters, namely: significant wave height; peak period; peak direction; 
directional spreading $\sigma$ (different from the JONSWAP peak spreading parameters); and JONSWAP's peak enhancement factor $\gamma$. Finally, a tracking procedure is applied in order to follow each event in time. If the peak frequency $f_{\mathrm{p}}$ of a tracked system exceeds $f_{\mathrm{s}}$ more than e.g. $70 \%$ of its total lifetime while similarly $\theta_{p}$ remains within $90^{\circ}$ from the local wind's direction, this system is definitely classified as a wind-sea (a swell otherwise). When the criterion on $f_{\mathrm{s}}$ as based on wind data (Eq. (26)) is found too sensitive - because wind stops blowing and so $f_{\mathrm{s}}$ increases too importantly - an empirical default value may be set in the tracking algorithm instead: here, it is taken as $f_{\mathrm{s}}=0.12 \mathrm{~Hz}$. This paper will demonstrate how this partitioning and tracking process allows a more accurate description and classification of either kind of wave systems.

\section{Results and discussion}

\subsection{Directional wave spectrum estimation over the array}

Figures 3 to 14 show the directional spectrum estimations - obtained with BMEM1 and IMLM1 - at the four buoy locations on four different dates. They respectively illustrate: 1/ a Westerly swell (Fig. 3-4, 05/02/10, 9am), 2/ a wind-sea-dominated sea state (Fig. 6-7, 14/02/10, 5am), 3/ a unidirectional bimodal sea (Fig. 9-10, 21/02/10, 10am), and 4/ a crossed bimodal sea (Fig. 12-13, 22/02/10, 7am). The corresponding estimates of variance spectral density $S(f)$ and directional Fourier coefficients $a_{1}(f), b_{1}(f), a_{2}(f)$ and $b_{2}(f)$ - without smoothing - are respectively plotted in Figures 5, 8, 11, and 14. Assuming wave stationarity (and neglecting any kind of measurement noise), the uncertainty upon sea state spectral estimates is related to the finiteness of measured samples, which is also known as sampling (or statistical, random...) error. In these figures, the $90 \%$ confidence interval limits of (scaled $X^{2}$-distributed) buoy A's spectral density estimates are added to the plots (see e.g. [35]). Were wave homogeneity ensured over the buoy array, all the buoys' spectral estimates should be consistent with such a statistical variability.

The directional spectrum of the first sea state (Fig. 3-5), estimated by all four buoys and both methods, is seen to be homogeneous over the spatial extension of the array, as a narrowbanded swell spectral shape with very similar spreading patterns. Only the mean and peak directions seem to vary over the array (about $30^{\circ}$ and $15^{\circ}$ respectively). This variation may be partly due to wave and tide interactions, as explained later on. The second sea state (Fig. 6-8) is made of the superposition of one westerly light swell and one strong north-easterly wind-sea. Buoys $A$ and $C$ - the southernmost ones (see Fig. 2) - identify the swell system whereas buoys $B$ and $D$ do not: the corresponding spectral densities in Figure 8 are almost unimodal (as seen on a linear scale) for the latter, indeed, which is not consistent with the theoretical sampling error based on buoy A's estimates. BMEM and IMLM spectral estimators yield similar directional spectra, yet they slightly mismatch on the directional location of the wind-sea's peak: it is found to lie between $20^{\circ}$ and $60^{\circ}$ (North-East) according to BMEM while it is homogeneously found close to $0^{\circ}$ (North) with IMLM. It is also noticed that the origin of the swell estimated from buoy $C$ does not exactly match that of the other buoys for IMLM (slightly West-North-West instead of West). The third sea state (Fig. 9-11) is composed of one developed wind-sea aligned with one long westerly swell - both of similar energy -, which make up together quite a unidirectional sea. This example illustrates the significant variability of the wind-sea's peak frequency estimation, which varies from $0.15 \mathrm{~Hz}$ to $0.19 \mathrm{~Hz}$ over the buoy array (i.e. peak periods varying from $5.3 \mathrm{~s}$ to $6.7 \mathrm{~s}$ ). In particular, the spectra estimated from buoys $C$ and $D$ exhibit a clear-cut separation between the swell and the wind-sea peaks, whereas the two systems appear very much closer at buoys $A$ and $B$. Again, a few inconsistencies are observed regarding statistical variability in Figure 11 wherein, for instance, the swell peak values vary more than expected according to theory under wave homogeneity assumptions over the whole buoy array. The variability of the wind- 
sea's peak frequency, however, seems explained by the sampling error. The fourth and last sea state (Fig. 12-14) also is bimodal but made of one swell from South-South-West and one wind-sea coming from North-North-East. Although there is good agreement between the buoys on the spectro-directional estimation of the wind-sea, the swell identification happens to be a bit trickier. The estimations from buoy $B$ permit to localize the swell as propagating from the peak direction $\sim 230^{\circ}$ (South-West). From buoy D, BMEM only reproduces a very much spread system with peak direction around $210^{\circ}$; IMLM is able to estimate a swell system consistent with buoy $B$ data however. Estimations from buoy $C$ are less precise: BMEM suffers from the major drawback raised in [1], where low-frequency system $180^{\circ}$ splitting occurs occasionally (Fig. 12). The westerly swell is split into two sub-systems - of same peak frequency -, the one almost from South and the second almost from North. IMLM does not split the system but rather produces swell directional shape with quite a large spread $\left(150^{\circ}\right.$-wide). Buoy $A$ hardly identifies the swell, which is not visible after directional estimation and spectro-angular smoothing. Here again, the variability of the spectral estimates related to the wind-sea is consistent with homogeneity assumptions. However, the variability found for estimates below $0.10 \mathrm{~Hz}$ may be higher than what theory predicts. Such an uncertainty on the low-frequency contents of the sea state emphasizes the limits of buoy measurements in the spectral estimation of long swell systems (even before tidal interactions issues are considered, see §5.2). This may be due to technical limitations (hydrodynamics, instrument noise, mooring interference...) but also default of stationarity/homogeneity of the wave field within the measurement area over about $17 \mathrm{~min}$ or recordings. It was observed, however (not illustrated here), that using no segment overlapping in the cross-spectral analysis increased significantly the occurrence of such directional artefacts in the sample.

About (frequency-dependent) directional estimates, a good agreement was mostly found between the four buoys, with more variability on the $2^{\text {nd }}$-order coefficients (see Fig. $5,8,11$, $\& 14)$. The monthly averaged value of the theoretical variance of the estimated $a_{1}, b_{1}, a_{2}$ and $b_{2}$ (see [36]) and the corresponding modulus $r_{1}=\left|c_{1}\right|^{2}$ and $r_{2}=\left|c_{2}\right|^{2}$ (Eq. (7-9)), given as

$$
\begin{aligned}
& \operatorname{Var}\left[a_{1}\right]=\frac{1}{2 v} \cdot\left[a_{1}^{2} z_{1}-2 a_{1} b_{1} b_{2}-a_{2}\left(2 a_{1}^{2}-1\right)+1\right] \\
& \operatorname{Var}\left[b_{1}\right]=\frac{1}{2 v} \cdot\left[b_{1}^{2} z_{1}-2 a_{1} b_{1} b_{2}+a_{2}\left(2 b_{1}^{2}-1\right)+1\right] \\
& \operatorname{Var}\left[a_{2}\right]=\frac{1}{v} \cdot\left(a_{2}^{2}-1\right)\left(r_{2}^{2}-1\right) \\
& \operatorname{Var}\left[b_{2}\right]=\frac{1}{v} \cdot\left(b_{2}^{2}-1\right)\left(r_{2}^{2}-1\right) \\
& \operatorname{Var}\left[r_{1}\right]=\frac{1}{2 v} \cdot\left[r_{1}^{2} z_{1}+\left(\frac{1}{r_{1}^{2}}-2\right)\left(2 a_{1} b_{1} b_{2}+a_{2}\left(a_{1}^{2}-b_{1}^{2}\right)\right)+1\right. \\
& \operatorname{Var}\left[r_{2}\right]=\frac{1}{v} \cdot\left(r_{2}^{2}-1\right)^{2}
\end{aligned}
$$

where $z_{1}=2\left(r_{1}^{2}-1\right)+\left(r_{2}^{2}-1\right) / 2$ (hats and frequency dependence have been omitted for convenience), is plotted against frequency in Figure 15. A general agreement is found over the array so that the directional estimation is quite similar for all buoys. Only buoy $C$ and buoy $D$ may exhibit slightly more variability than buoys $A$ and $B$ : this is observed in particular for coefficients $b_{1}(f), a_{2}(f)$ and $b_{2}(f)$ in Figures $15(\mathrm{~b}-\mathrm{d})$. The averaged variance of $\left(a_{1}(f), b_{1}(f)\right)$, on the one hand, and $\left(a_{2}(f), b_{2}(f)\right)$, on the other hand, look very similar when compared side 
by side. However, the variance of the $2^{\text {nd }}$-order coefficients clearly is found greater than that of $1^{\text {st }}$-order coefficients, which confirms the visual inspection of Figures $5,8,11, \& 14$. This subsequently illustrates the fact that $a_{1}(f)$ and $b_{1}(f)$ are generally more accurately estimated and therefore, more reliable - than $a_{2}(f)$ and $b_{2}(f)$ from buoy measurements. Also, it is observed that the $1^{\text {st }}$ - and $2^{\text {nd }}$-order coefficients variability is greater for $f<0.07 \mathrm{~Hz}$ and $\left.f\right\rangle$ $0.35 \mathrm{~Hz}$ respectively, which means that $\left(a_{1}(f), b_{1}(f)\right)$ and $\left(a_{2}(f), b_{2}(f)\right)$ are less accurately estimated in the low- and high-frequency range respectively. For both orders, the most accurate estimations are obtained around $0.18 \mathrm{~Hz}$ (i.e. in the range $[0.15 \mathrm{~Hz} ; 0.25 \mathrm{~Hz}]$ ) and a second best estimation range is observed around $0.09 \mathrm{~Hz}$. This must be related to the relative occurrence of the systems crossing the array as measured by the buoys: the distribution of the overall peak frequency $f_{\mathrm{p}}$ (Eq. (21)) estimated from each buoy is depicted in Figure 16. The histogram $\left(0.01 \mathrm{~Hz}\right.$-wide bins) shows that sea states with $f_{\mathrm{p}}$ around $0.08 \mathrm{~Hz}$ and within $[0.16 \mathrm{~Hz} ; 0.20 \mathrm{~Hz}]$ - respectively standing for swell and wind-sea systems - have the greatest occurrence frequency at this location. The statistical variability of the directional estimation may therefore be directly related to the level of energy measured within a given frequency band, which conditions the relative cross-spectral noise level. Were always seas unimodal, for instance, Figure 15 would probably exhibit slightly different variance curves (probably more U-shaped ones). Let us stress that, as the estimated Fourier coefficients have been used to figure out the statistical variability related to each of them (Eq. (27)), other sources of estimation error are also likely to be included in these results, namely: buoy calibration; measurement noise; buoy response (accelerometers' transfer functions and filtering); crossspectral analysis, etc. (see e.g. [6]). From a more general point of view, the directional measurements performed by SEAWATCH Mini II buoys are found satisfactorily reliable - up to the $2^{\text {nd }}$ order - in the approximate range $[0.08 \mathrm{~Hz} ; 0.30 \mathrm{~Hz}]$, which matches the WADIC project's conclusions [37] regarding systems like MAREX and WAVETRACK for the determination of the mean direction and spreading per frequency band.

\subsection{Overall parameters estimations}

Figures $17(\mathrm{a}-\mathrm{f})$ depict the evolution of overall parameters $f_{\mathrm{p}}, H_{\mathrm{m} 0}, \theta_{\mathrm{m}}, \sigma_{\mathrm{m}}$, and $\Lambda$ estimated with the four buoys during the month of February 2010 using BMEM1 (IMLM1 yielded very similar results). Wind and tidal data also are included in the plots, as separation frequency (Eq. (26), Fig. 17(a)), mean wind speed (divided by two, Fig. 17(b)), mean wind direction (Fig. 17(c)) and tidal level, speed and direction (Fig. 17(f)). Three particular windows are highlighted in Figure 18, which respectively cover the periods $5^{\text {th }}-7^{\text {th }}, 13^{\text {th }}-17^{\text {th }}$ and $21^{\text {st }}-23^{\text {rd }}$ of February (examples illustrated in Fig. 3 to 14 are taken out from these windows). Each period describes a different sea state event, possibly changing from unimodality to bimodality and conversely, according to the $f_{\mathrm{p}}$ plots (Subfigs. (1a), (2a) \& (3a)). Window 1 (2 days) corresponds to a clear sequence of swell-dominated sea states. Window 2 (4 days) illustrates a sequence of wind-sea-dominated sea states until the $16^{\text {th }}$ of February, after which the sea state becomes mixed (swell + wind-sea). Window 3 (2 days) is more varied: it successively exhibits a mixed sea, a wind-sea growth phase covering a weak swell and, eventually, a sequence of swell-dominated sea states.

The superposition of the plots of estimated peak frequency for all buoys (Fig. 17(a)) - prior to inspecting the wave systems tracking results (\$5.3) - already provides some precious information about the contents of the sea states. The wave climate is alternatively made of swell- and wind-sea-dominated sequences, with some bimodal mixed sea conditions such as those observed between the $20^{\text {th }}$ and the $22^{\text {nd }}$ of February, where the two wave systems - of similar energy - are alternatively leading the sea state: the peak frequency estimate skips from the swell to the wind-sea component and conversely. The superposition of the buoy results permits the clear identification of the actual bimodality of this sea state sequence, indeed. The mean significant wave height is close to $2 \mathrm{~m}$ and ranges from $1 \mathrm{~m}$ to $6 \mathrm{~m}$ through the month of February (Fig. 17(b)). Parameters $\theta_{\mathrm{m}}, \sigma_{\mathrm{m}}$, and $\Lambda$ (Fig. 17(c-e)) vary according to the contents of the sea states but also seem to constantly oscillate in phase with tide (Fig. 
17(f)). In particular, $\theta_{\mathrm{m}}$ amplitudes are found slightly different according to the buoys (Buoy $\mathrm{C}$ exhibiting the largest oscillations indeed). An estimation of the spectral density of these three parameters' time-series after trend-removal by moving average filtering has been realised (not depicted here by lack of space), which revealed that the three time-series $-\theta_{\mathrm{m}}, \sigma_{\mathrm{m}}$, and $\Lambda$ - have a peak frequency corresponding to periods of $\sim 12.30 \mathrm{~h}, \sim 6 \mathrm{~h}$ and $\sim 12.30 \mathrm{~h}$ respectively. Accordingly, the variations of these wave properties are, at least in part, related to tidal currents. A comparison between the three windows in Figure 18(c-f) illustrates this influence clearly. The mean direction of waves $\theta_{\mathrm{m}}$ tends to move to a more Northerly direction during the flood rising (from the direction $230-240^{\circ}$ ) and, conversely, becomes more Southerly with ebb. The mean spreading parameter is affected in a different way: $\sigma_{\mathrm{m}}$ increases with the current (ebb or flood) and reaches a local minimum during slack waters, so that the mean spreading seems related to the magnitude of the current rather than its direction. Finally, the spectral bandwidth $\Lambda$ follows a somewhat more subtle evolution: when waves are westerly (window 1), the bandwidth is found to decrease when the ebb sets in (from N-E, $\sim 50^{\circ}$ ); then, in case of northerly waves (window 2), bandwidth increases in ebbs. It must be concluded that bandwidth $\Lambda$ is reduced in opposing currents whereas it is magnified in following currents. Let us also point out that bandwidth variations are found particularly more important when the wind-sea component is significant (windows 2\&3). If one considers significant wave height, some clear sensitivity to tide is noticed during the wind-sea-dominated sequence around the $14^{\text {th }}$ of February in window 2 (Fig. 18(2b)). In a general manner, therefore, the oscillation of the observed wave parameters seem more important when the wind-sea is significant compared to the possible coexisting swell(s). This observation shall be examined after using the wave system identification and time tracking algorithms (§5.3).

At this stage, some basic knowledge related to interactions between large scale, slowly timeand space- varying currents as tides and ocean waves are needed for a better analysis of these results. The Doppler-shifted dispersion equation is (see e.g. [38])

$\left(\omega-k U_{t} \cos \psi\right)^{2}=g k \tanh (k h)$

where $U_{t}$ is the (depth-integrated) tidal flow speed (in $\mathrm{m}^{-1} \mathrm{~s}^{-1}$ ) and $\psi$ denotes the smallest angular distance between the tide's and waves' direction (in radians). If $U_{t}=0$ (no current), the classical dispersion relationship is obtained. In deep water, it may be shown that the wave-number is typically modified by a factor $4^{*}\left(1+\sqrt{ }\left(1+4 U_{t} \cos \psi / c\right)\right)^{-2}$, where $c$ is the phase celerity of (apparent) waves. Thus, the current's effect on waves found more important when the ratio $U_{t} / c$ is large, that is, for shorter waves (and/or strong currents). Waves are modified by Doppler effect and interactions with tide simultaneously, which are lengthened (shortened) in a following (an opposing) current accordingly. In terms of wave dynamics, it may be theoretically shown [39] that an opposing current increases the local height of waves whereas it is reduced by the wave lengthening operated by a following current. Refraction may also occur when short directional waves meet a current and thus modify their direction. The observed variations of (overall) wave parameters due to tide are therefore fully expected and partly explained.

Finally, the space variability across the four buoys array of the overall parameters over the month of February 2010 is summarized in Table 2. The mean variations of parameters $H_{\mathrm{m} 0}$, $\Lambda, \theta_{\mathrm{p}}, \theta_{\mathrm{m}}$ and $\sigma_{\mathrm{m}}$ are calculated as the monthly average value of the largest difference between the parameters estimated over the array in each sea state, that is,

$\overline{\Delta p}=\frac{1}{N} \cdot \sum_{i=1}^{N}\left[\max \left(p_{i, j}\right)_{1 \leq j \leq 4}-\min \left(p_{i, j}\right)_{1 \leq j \leq 4}\right]$ 
$p_{\mathrm{i}, \mathrm{j}}$ standing for any of the parameters $H_{\mathrm{m} 0}, \Lambda$ and $\sigma_{\mathrm{m}}$ at instant $i$ and for buoy $j, N$ denoting the total number of measurements in the month, and similarly,

$$
\overline{\Delta \theta}=\frac{1}{N} \cdot \sum_{i=1}^{N} \max \left(\operatorname{diff}\left(\theta_{i, j}, \theta_{i, k}\right)_{\substack{1 \leq j, k \leq 4 \\ j \neq k}}\right)
$$

for any of $\theta_{\mathrm{p}}$ and $\theta_{\mathrm{m}}$, where $\operatorname{diff}\left(\theta_{\mathrm{i}, \mathrm{j}}, \theta_{\mathrm{i}, \mathrm{k}}\right)$ denotes the minimal angular distance (in degrees) between $\theta_{\mathrm{i}, \mathrm{j}}$ and $\theta_{\mathrm{i}, \mathrm{k}}$ on the compass, at instant $i$ for buoys $j$ and $k$ respectively. The variations are given for BMEM1 and IMLM1. It is observed that the directional spreading variations generally are small $\left(<6^{\circ}\right.$ on average in each sea state). However, larger variations are obtained for peak and mean wave directions, as $\sim 33^{\circ}$ and $\sim 20^{\circ}$ respectively. The space variations of spectral parameters like $H_{\mathrm{m} 0}$ and $\Lambda$ are not affected by the estimation method, as expected from their definitions (Eq. (20) \& (25)) (very little influence of smoothing). Yet, it is interesting to note that, while the estimation of the significant wave height may vary about $12 \%$ within the array, the mean variations of the spectral bandwidth figure are about $0.03 \mathrm{~Hz}$, which is not negligible since $\Lambda$ is found to range from 0.04 to $0.35 \mathrm{~Hz}$ with a mean value of $0.15 \mathrm{~Hz}$ during the same month (i.e. $\sim 20 \%$ on average).

\subsection{Wave systems time tracking}

Figure 19 depicts the time-evolution of the peak frequency (a), significant wave height (b), peak direction (c) and directional spreading (d) of all individual wave systems identified and tracked over the array from directional spectra estimated using BMEM2. In general, the variability of the peak enhancement factor $\gamma$ (not plotted here, but observed to vary in the range $[1 ; 6]$ ) meant that no specific comment can be made regarding the spectral bandwidth of each individual wave system. In [1], a spectral bandwidth figure for individual wave systems was formed as the distance $(\mathrm{Hz})$ separating the high and low $10 \mathrm{~dB}$-peak-attenuation cut-off frequencies, which was found very much correlated to the individual system's peak frequency. This parameter is not represented in Figure 19 however, for it did not bring any significant information to the present purpose. Once again, tidal data are given in Figure 19(e). Swell/wind-sea separation frequency, mean wind speed (divided by two) and mean wind direction are added to Figures 19(a-c) in the same manner as Figures 17(a-c). These charts are obtained as the superposition of the tracking plots derived from each buoy independently, so that the resulting wave system information is considerably richer. Swells are plotted as dots ('.') whereas wind-seas are denoted by stars ('*'). The classification into either type of system has been realised thanks to the time tracking algorithm described in $\S 4.2$. These figures can be compared to Figures $17(\mathrm{a}-\mathrm{d})$ so that the significant refinement brought up by the wave system identification and tracking procedure may be emphasized. The individual swells and wind-seas evolution is clearly distinguishable in Figure 19(a), where slow peak frequency increases in the low-frequency range $(<0.1 \mathrm{~Hz})$ coexist with rapid peak frequency drops from high-frequency (up to $0.4 \mathrm{~Hz}$ ) corresponding to wind-sea growths, which are found consistent with wind data. Indeed, the properties of the identified wind-sea systems are in good agreement with separation frequency, wind strength and direction data. The particular growing sea episode occurring around the $8^{\text {th }}$ of February - which could not be observed from the overall characterisation (Fig. 17(a)) - highlights the strong influence of tide upon the wind-sea's properties, peak frequency being the most illustrative parameter. More generally, tide is found influential mostly on parameters related to wind-seas (see e.g. Fig. $19(a-b)$ for $H_{m 0}$ on the $11^{\text {th }}-15^{\text {th }}$ of February), including directional spreading (Fig. 19(d)). Swell-related properties seem much less affected by the tidal cycle except peak direction $\theta_{p}$ - which sometimes oscillates strongly $\left(11^{\text {th }}-15^{\text {th }}\right.$ of February, Fig. 19(c)) - and, to some extent, directional spreading $\sigma$ (see e.g. Fig. 19(d) around the $25^{\text {th }}$ of February). The directional origin of swell and wind-sea systems identified during February 2010 from buoy $A$ measurements along with that of wind and tide are plotted as polar histograms in Figure 20. 
Swells are shown to arise essentially from West whereas the origin of wind-seas is regularly distributed from West to North-East - which is consistent with the wind's origin and fetch limitations due to the proximity to the coast - with but two slightly more represented sectors of higher fetch (West and North-East). Interestingly, in this location, wave systems, tidal flows and wind are found to occur mostly within these two very sectors. The parameters variations over a shorter time interval using the windows defined previously in Figure 18 are illustrated in Figure 21 where the effect of tide upon the wave system parameters is highlighted. The swell peak frequency is not influenced by tide at all, as expected from theory. For wind-seas, however, this influence is really strong and particularly visible in window 2 (2a) from the $13^{\text {th }}$ to the $15^{\text {th }}$ of February. It is seen that peak frequency is reduced in opposing currents and vice versa, which apparently contradicts theory (high-frequency spectral contents should be enhanced, and so $f_{\mathrm{p}}$ would be expected to increase). In fact, an opposing current also filters out high-frequency waves (through wave breaking/white-capping effect) while non-linear wave-wave interactions may occur, which results in a reduction of peak frequency and bandwidth. The evolution of $H_{\mathrm{mo}}$ for swells and wind-sea must be regarded with some care, for the partitioning may introduce some noise into this information. However, it seems clear from window $2(2 b)$ that tidal currents increase the wind-sea's energy when opposing it and conversely, while wind modulates the mean wave height on a longer time scale. The energy variations of swells are less easy to appreciate, however. In window 3 , the sea states start as bimodal and unidirectional (in line with the flood, from $240^{\circ}$ ) until wind suddenly turns back by about $180^{\circ}$ to $\sim 80^{\circ}$ so that a new unidirectional bimodal sea state settles down, as opposite swell and wind-sea systems propagating against each other. Strong non-linear wave-wave interactions are expected to occur then (all the more as the ratio of the peak frequencies is close to half/double, which facilitates the energy transfer between wave components) which may explain the increase of energy in the swell system observed during the $22^{\text {nd }}$ of February. The evolution of wind-sea peak direction is found consistent with theory, i.e. waves generally align themselves with the flood/ebb direction (2c\&3c). For swells, the opposite is observed, but one should be cautious to this respect because of the larger directional uncertainty in low-frequency wave buoy measurements, as seen in \$5.1. About directional spreading, the same observation as for the overall parameter may be made - for both kinds of system - , which is the strong sensitivity to water circulation with minimum spreading at slack tides.

\section{Conclusions}

This paper has addressed the directional analysis of sea states occurring near the Wave Hub test facility for wave-energy devices located in the South-West of Great-Britain (Cornwall). The study was based on the processing of one full month of data (February 2010), from a unique array of four time synchronized SEAWATCH Mini II directional buoys.

The results have firstly shown the extremely good quality of the data provided by the buoy array deployed over a relatively small spatial extent $\left(\sim 500 \mathrm{~m}^{\star} 500 \mathrm{~m}\right)$ : a very satisfactory agreement has been found on the estimation of overall wave parameters as well as on the wave system identification performed from the buoys' data. In particular, the remarkable correlation of wave systems characteristics to the properties of the local wind - which proves the relevance of the spectro-directional partitioning in metocean and ocean engineering studies - must be underlined.

This study involved two methods for the estimation of the directional distribution function, namely BMEM and IMLM (MEP having exhibited very similar distribution functions to BMEM after spectro-angular smoothing). A general agreement between the methods has been obtained on the resulting spectro-directional shapes, even though some occasional differences of sensitivity were noticed in the low-frequency range $(f<0.1 \mathrm{~Hz})$. The BMEM's peak-splitting propensity was noticed in a few cases while IMLM produced somewhat broader directional shapes. 
Furthermore, it has been seen that the spectral estimates derived from about 17 minutes of recording could sometimes exhibit some variability in space, particularly below $0.1 \mathrm{~Hz}$. It is yet not clear whether the low-frequency accuracy of the directional buoys (possibly allied with their sensitivity to tidal flow) is in the first place responsible for this variability, or whether the validity of stationarity and homogeneity of the water elevation process assumed over the array might not be fully ensured for the present measurement set-up (array size and recording time). First-order directional Fourier coefficients $\left(\hat{a}_{1}(f), \hat{b}_{1}(f)\right)$ were found to agree well over the buoy array whereas second-order estimates $\left(\hat{a}_{2}(f), \hat{b}_{2}(f)\right)$ exhibited greater variability. The average statistical variability of $a_{1}(f), b_{1}(f), a_{2}(f)$, and $b_{2}(f)$ has been found to be very similar for all four buoys, which proves again the coherence of the measurement array. The most reliable buoy directional estimations are theoretically realized within $[0.15 \mathrm{~Hz} ; 0.25 \mathrm{~Hz}]$ in this particular wave climate (Fig. 15) so that the variability of the lowfrequency directional estimation referred to here above may also be partly explained by the less accurate Fourier coefficients estimations (higher relative noise level).

This study has also revealed the strong influence of tide upon the estimated wave parameters. From a general point of view, mean characteristics of sea states such as spectral bandwidth, mean direction and mean directional spreading were found to be correlated to the local tide data, all the more as the sea state is wind-sea-dominated. In particular, spectral bandwidth $\Lambda$ and mean wave direction $\theta_{\mathrm{m}}$ appeared to vary in phase with the tidal cycle most of the time whereas mean directional spreading $\sigma_{\mathrm{m}}$ was only sensitive to water circulation, as systematically larger during flood and ebb phases and minimal at slack tides ( $6 \mathrm{~h}$ cycles). The spectral significant wave height $H_{\mathrm{mo}}$ has exhibited a small but manifest correlation to tide within the considered data set, especially in wind-sea-dominated seas. A general good agreement has been obtained between field measurements and theory on wave and currents interactions.

The spectro-directional partitioning and time-tracking of identified wave systems has enabled a better analysis of the tidal effect upon the sea state contents. Thus, the peak direction and directional spreading of estimated swell and wind-sea systems have been found equally influenced by the tide, although these observations must be put into perspective for swells due to the low-frequency estimation uncertainty mentioned above. Peak frequency and significant wave height of wind-seas also have been found strongly correlated to the tidal variations - much smaller or no correlation at all has been found for swell components - , so that wind-seas generally are proved responsible for the overall sensitivity of sea states to tide over the considered area, as expected from theory.

This study therefore constitutes a first interesting and exploratory insight into the analysis of wave/current interactions from extended in situ observations using a buoy array. In terms of WEC response, this study has highlighted the need to consider the combined effect of wave and tidal flows to understand the response and efficiency of the devices for the purposes of evaluating the extractable energy to greater accuracy. The importance of considering wave systems separately within a sea state must be recalled, and in particular, the fact a set of overall parameters - though straightforward to compute (e.g. $\left.H_{\mathrm{m} 0}, f_{\mathrm{p}} \ldots\right)$ - are not sufficient (not to say misleading) to describe the actual sea complexity, i.e. its possible multimodality. Swell and wind-sea components, with their own characteristics (energy, peak direction and frequency... which make physically sense at the scale of an individual unimodal wave system), have separate effects on moored ocean structures like WECs. According to the technology (and the mooring system) indeed, swell or wind-sea may influence more specifically energy extraction performance, device orientation, low-frequency response, fatigue etc. A deep understanding of the interactions between wind, tidal currents and wave systems - and their implications on the local wave characteristics - is crucial for the optimisation of wave-energy harnessing as well as offshore and coastal engineering studies in general. An acoustic Doppler current profiler is currently being installed within the buoy array in view of better characterising these interactions in the future. In parallel, spectral 
wave numerical simulations are being carried out to support the present observations near the Wave Hub test facility.

\section{Acknowledgements}

The authors would like to express their thanks to the European Union for funding this work. The buoy array and its operation were funded through the ERDF, PRIMaRE project whilst EQUIMAR project (FP7 grant agreement FP7-213380) funded staff to participate on the research. We also gratefully acknowledge the UK Meteorological Office for providing modelled wind data for the region.

\section{References}

[1] J.-B. Saulnier, M. Prevosto, C. Maisondieu, Refinements of sea states statistics for Marine Renewables: a case study from simultaneous buoy measurements in Portugal, Renewable Energy 36 (2011), 2853-2865.

[2] M. J. Tucker, Recommended standard for wave data sampling and near-real-time processing, Ocean Eng. 20 (1993) 459-474.

[3] M.S. Longuet-Higgins, D.E. Cartwright, N.D. Smith, Observations of the directional spectrum of sea waves using the motions of a floating buoy, in: Ocean Wave spectra, Prentice-Hall, Inc., Englewood Cliffs, New Jersey, USA, 1963, pp. 111-136.

[4] M. Isobe, K. Kondo, K. Horikawa, Extension of MLM for estimating directional wave spectrum, in: Proc. Symp. on Description and modelling of Directional Seas, Lyngby, Denmark, Tech. University, A6, 1984, pp. 1-15.

[5] M. Benoit, P. Frigaard, A. Schäffer, Analysing multidirectional wave spectra: a tentative classification of available methods, In: Proc. $27^{\text {th }}$ IAHR Congress, Seminar on multidirectional waves and their interaction with structures, San Francisco, USA, 1997, pp. 131-158.

[6] A.J. Kuik, G.P. van Vledder, L.H. Holthuijsen, A method for the routine analysis of pitch-and-roll buoy wave data, J. Phys. Oceanogr. 18 (1988) 1020-1034.

[7] M.J. Tucker, Interpreting directional data from large pitch-roll-heave buoys, Ocean Eng. 16 (1989) 173-192.

[8] M.A. Arribas, J.J. Egozcue, Normalized maximum-likelihood estimators of the directional wave spectrum, J. Atmos. and Ocean. Tech. 12 (1994) 668-673.

[9] A.J. Chadwick, D.J. Pope, J. Borges, S. Ilic, Shoreline directional wave spectra, Part 1: An investigation of spectral directional analysis techniques, in: Proc. Instn. of Civ. Engrs. in Wat., Marit. and Energy, vol. 112, n³, 1995, pp. 198-208.

[10] P.D. Welch, The use of fast Fourier transform for the estimation of power spectra: a method based on time averaging over short, modified periodograms, in: I.E.E.E. Trans. Audio and Electroacoust., AU-15, 1967, pp. 70-73.

[11] L.E. Borgman, Estimation of wave directional spectra from pitch-roll buoy data, J. Waterways, Port., Coastal and Ocean Eng. 120 (1969) 93-115.

[12] H.E. Krogstad, S.F. Barstow, O. Haug, D.J.H. Peters, Directional distributions in wave spectra, in: Proc. Int. Conf. on Ocean Wave Measmts. and Anal. (WAVES'97), 2, Virginia Beach, Virginia, USA, 1997, pp. 883-895.

[13] M. Benoit, Practical comparative performance survey of methods used for estimating directional wave spectra from heave-pitch-roll data, in: Proc. $23^{\text {rd }}$ Int. Conf. on Coast. Eng. (ASCE), Venice, Italy, 1992, pp. 62-75.

[14] J. Van Heteren, Estimation of multi-modal directional wave spectra from tri-orthogonal measurements, Coastal Eng. 7 (1983) 205-231. 
[15] J. Capon, High-resolution frequency -wave-number spectrum analysis, in: Proc. I.E.E.E., vol. 57, 1969, pp. 1408-1418.

[16] H.E. Krogstad, Maximum likelihood estimation of ocean wave spectra from general arrays of wave gauges, Modeling, Identification and Control 9 (1988) 81-97.

[17] J. Oltman-Shay, R.T. Guza, A data-adaptative ocean wave directional-spectrum estimator for pitch-roll type measurements, J. Phys. Oceanogr. 14 (1984) 1800-1810.

[18] O.U. Nwogu, Maximum entropy estimation of directional wave spectra from an array of wave probes, Appl. Ocean Res. 11 (1989) 176-182.

[19] S.R. Massel, R.M. Brinkman, On the determination of directional wave spectra for practical applications, Appl. Ocean Res. 20 (1998) 357-374.

[20] A. Lygre, H.E. Krogstad, Maximum entropy estimation of the directional distribution in ocean wave spectra, J. Phys. Oceanogr. 16 (1986) 2052-2060.

[21] O. Haug, H.E. Krogstad, Estimation of directional spectra by ML/ME methods, in: Proc. Conf. on Ocean Wave Measurement and Analysis, New Orleans, USA, 1994, pp. 394-405.

[22] R.F. Marsden, B.-A. Juszko, An eigenvector method for the calculation of directional spectra from heave, pitch and roll buoy data, J. Phys. Oceanogr. 17 (1987) 21572167.

[23] N. Hashimoto, K. Kobune, Estimation of directional spectra from the maximum entropy principle, in: Proc. $5^{\text {th }}$ Int. Offshore Mech. and Arct. Eng. Symp., Tokyo, Japan, vol. 1, 1986, pp. 80-85.

[24] J.P. Burg, Maximum entropy spectral analysis, PhD Thesis Report, Stanford University, USA, 1975.

[25] M.D. Earle, K.E. Steele, D.W.C. Wand, Use of advanced directional wave spectra analysis methods, Tech. note, Ocean Eng. 26 (1999) 1421-1434.

[26] J. Portilla, F.J. Ocampo-Torres, J. Monbaliu, Spectral partitioning and identification of wind sea and swell, J. Atmos. and Ocean Tech. 26 (2009) 107-122.

[27] R.B. Blackman, J.M. Tukey, The measurement of power spectra, Dover Publications Inc., New-York, USA, 1959.

[28] J.-B. Saulnier, Refined wave climatology as a contribution to the design of wave energy conversion systems, Ph.D. Thesis Report, Ecole Centrale de Nantes, France, Instituto Superior Técnico, Lisbon, Portugal, 2009.

[29] C. Guedes Soares, Representation of double-peaked sea wave spectra, Ocean Eng. 11 (1984) 185-207.

[30] M.-A. Kerbiriou, M. Prevosto, C. Maisondieu. Influence of an improved sea-state description on a wave energy converter production, in: Proc. $26^{\text {th }}$ Int. Conf. on Offshore Mech. and Arct. Eng., San Diego, California, USA, 2007.

[31] J.L. Hanson, O.M. Phillips, Automated analysis of ocean surface directional wave spectra, J. Atmos. and Ocean Tech. 18 (2001) 277-293.

[32] M.D. Earle, Development of algorithms for separation of sea and swell, National Data Buoy Center Tech. Rep. MEC-87-1, 1984.

[33] J.E. Aarnes, H.E. Krogstad, Partitioning sequences for the dissection of directional Ocean Wave Spectra : a Review, Tech. Rep. E.U. ENVIWAVE Research Programme (EVG-2001-00017, Wp4), 2001.

[34] K. Hasselmann, T.P. Barnett, E. Bouws, H. Carlson, D.E. Cartwright, K. Enke, J.A. Ewing, H. Gienapp, D.E. Hasselmann, P. Kruseman, A. Meerburg, P. Mueller, D.J. Olbers, K. Richter, W. Sell, H. Walden, Measurements of wind-wave growth and swell decay during the Joint North Sea Wave Project (JONSWAP), Deutsche Hydr. Zeit. 81 A12 (1973) 1-95.

[35] D. R. Brillinger, Time series, data analysis and theory, in: Holt, Rinchart and Winston, New York, 1975.

[36] R.B. Long, The statistical evaluation of directional spectrum estimates derived from pitch/roll buoy data, J. Phys. Oceanogr. 10 (1980) 944-952.

[37] J. Allender, T. Audunson, S.F. Barstow, S. Bjerken, H.E. Krogstad, P. Steinbakke, L. Vartdal, L.E. Borgman, C. Graham, The WADIC project: A comprehensive field evaluation of directional wave instrumentation, Ocean Eng. 16 (1989) 505-536. 
[38] I.G. Jonsson, Wave-current interactions, in: B. Le Méhauté, D.M. Hanes (Eds.), The Sea, 1990, pp. 65-120.

[39] N. E. Huang, D.T. Chen, C.-C. Tung, J.R. Smith, Interactions between steady nonuniform currents and gravity waves with applications for current measurements, J. Phys. Oceanogr. 2 (1972) 420-431. 


\begin{tabular}{lcccc}
\hline Characteristics & $\boldsymbol{A}(\mathbf{5 2})$ & $\boldsymbol{B}(\mathbf{4 9})$ & $\boldsymbol{C}(\mathbf{5 0})$ & $\boldsymbol{D}(\mathbf{5 1 )}$ \\
\hline Mean location & $50^{\circ} 18^{\prime} 28^{\prime \prime} \mathrm{N}$, & $50^{\circ} 18^{\prime} 35^{\prime \prime} \mathrm{N}$, & $50^{\circ} 18^{\prime} 18^{\prime \prime} \mathrm{N}$, & $50^{\circ} 18^{\prime} 40^{\prime \prime} \mathrm{N}$, \\
Mean water depth & $5^{\circ} 39^{\prime} 47^{\prime \prime} \mathrm{W}$ & $5^{\circ} 40^{\prime} 21^{\prime \prime} \mathrm{W}$ & $5^{\circ} 40^{\prime} 13^{\prime \prime} \mathrm{W}$ & $5^{\circ} 39^{\prime} 58^{\prime \prime} \mathrm{W}$ \\
Failure rate & $37.05 \mathrm{~m}$ & $37.50 \mathrm{~m}$ & $38.15 \mathrm{~m}$ & $40.75 \mathrm{~m}$ \\
Feb. 2010) & $1 / 1343(<0.1 \%)$ & $1 / 1343(<0.1 \%)$ & $1 / 1343(<0.1 \%)$ & $1 / 1343(<0.1 \%)$ \\
\hline
\end{tabular}

Table 1: Characteristics of the directional buoys deployed near the Wave Hub test site (February 2010). 


\begin{tabular}{lcc}
\hline Variations & BMEMI & IMLMI \\
\hline$\overline{\Delta H_{m 0}}$ & $0.26 \mathrm{~m}(12.2 \%)$ & $0.25 \mathrm{~m}(11.8 \%)$ \\
$\overline{\Delta \Lambda}$ & $0.03 \mathrm{~Hz}$ & $0.03 \mathrm{~Hz}$ \\
$\frac{\Delta \theta_{p}}{\overline{\Delta \theta_{m}}}$ & $35.1^{\circ}$ & $31.6^{\circ}$ \\
$\frac{\Delta \sigma_{m}}{19.9^{\circ}}$ & $5.8^{\circ}\left(12.6^{\circ} \%\right)$ & $20.2^{\circ}$ \\
\hline
\end{tabular}

Table 2: Mean space variability of overall wave parameters obtained with BMEM1 and IMLM1 over the measurement buoy array (Eq. (29)\&(30)) during February 2010. 


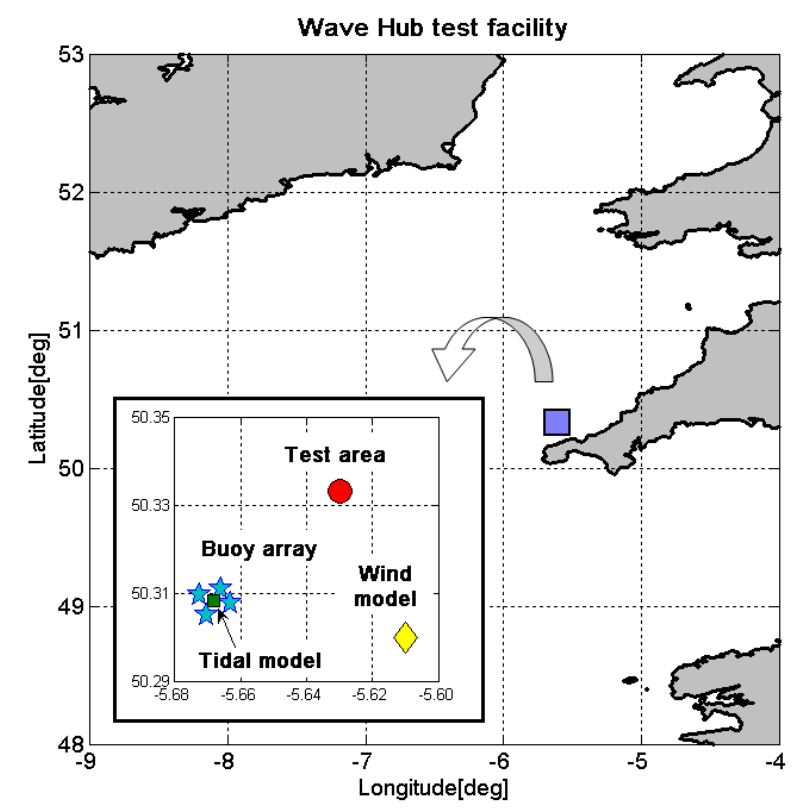

Fig. 1. Location of the Wave Hub test area and measurement buoys array (Cornwall, UK), wind (UK MetOffice unified model) and POLPRED ${ }^{\odot}$ tidal model grid-point locations. 


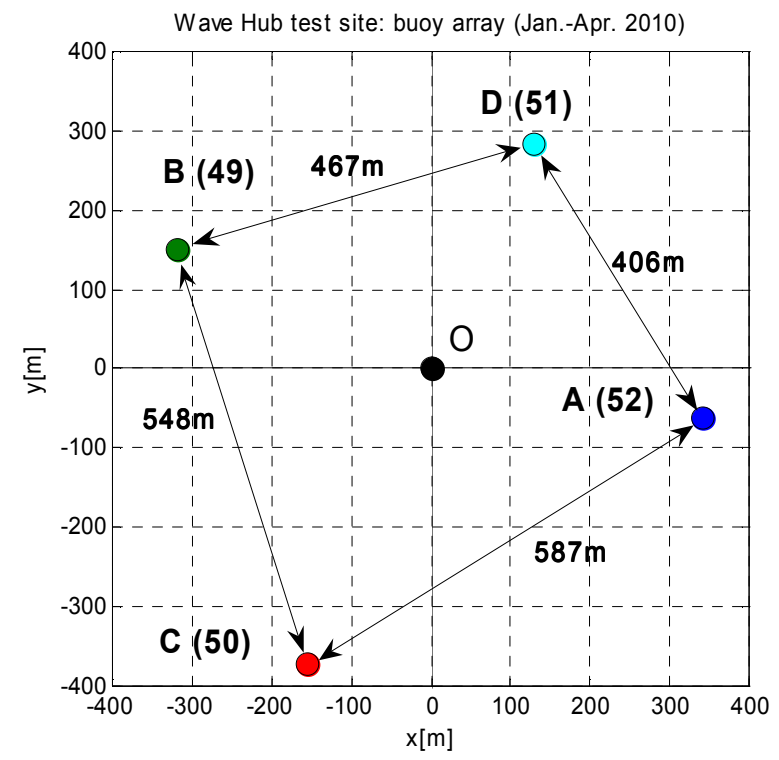

Figure 2: Measurement buoys array configuration in the Cartesian plane during the period January to April 2010. 

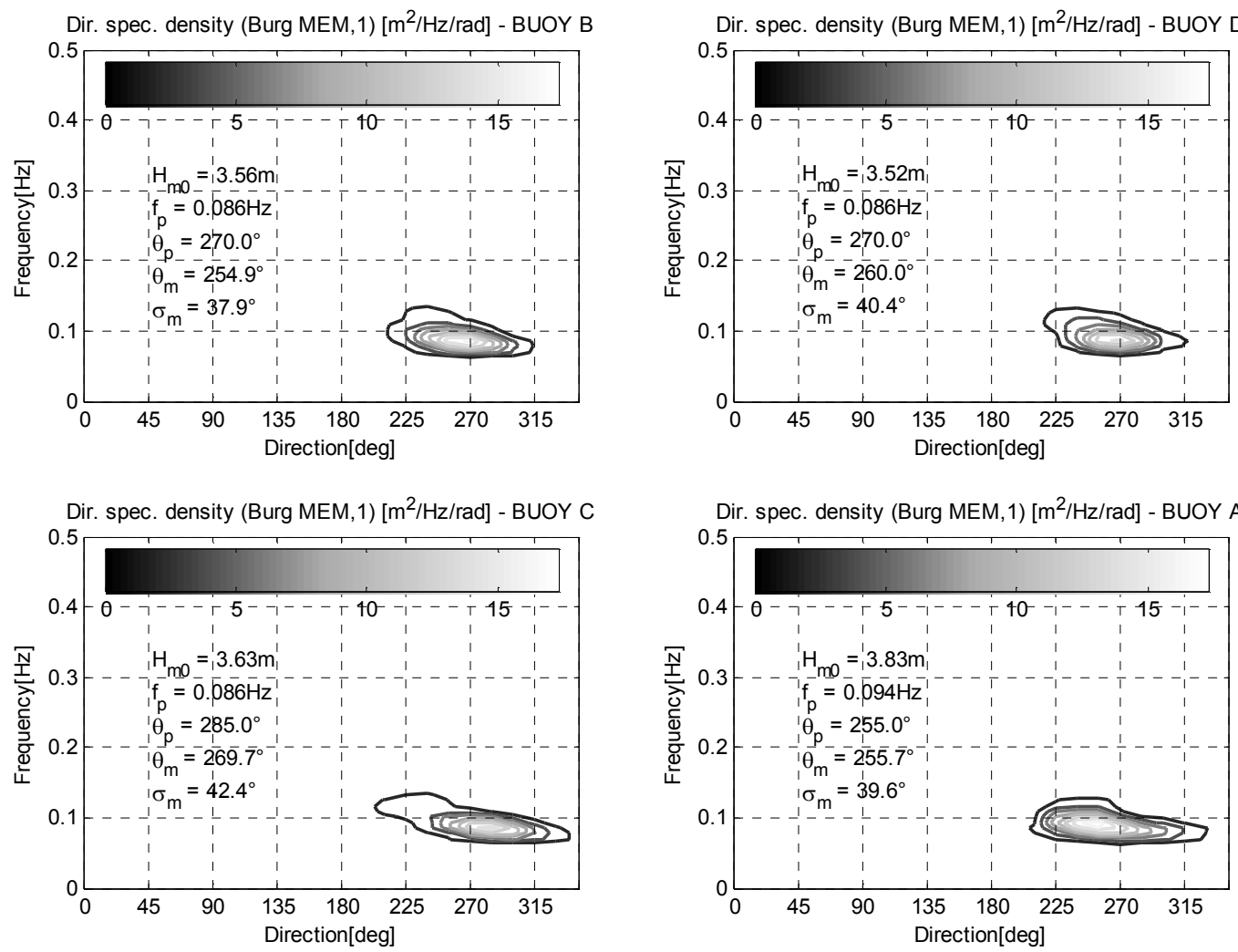

Figure 3: Directional spectra estimated with BMEM1 at the four measurement buoy locations on the $5^{\text {th }}$ of February 2010, 9am. 

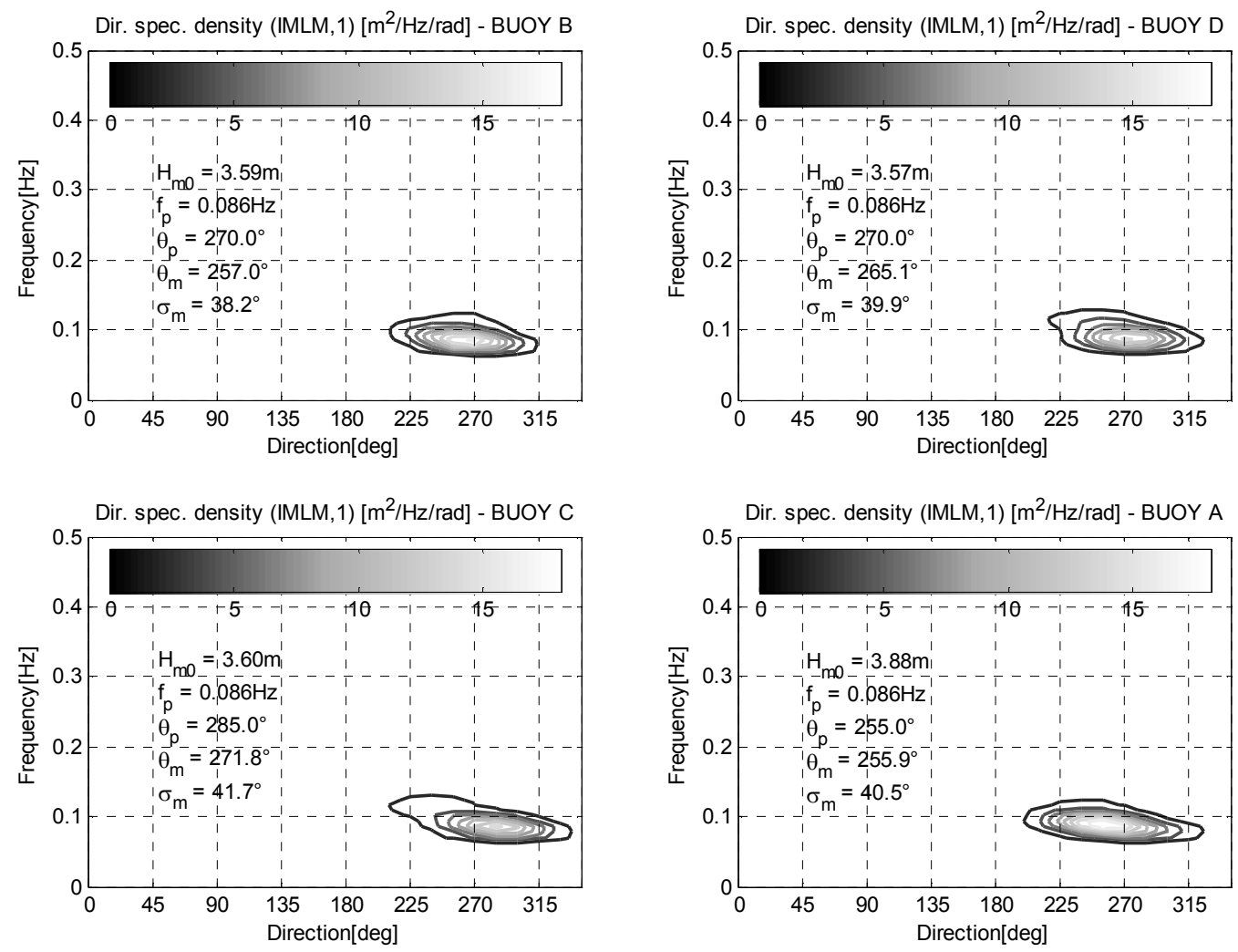

Figure 4: Idem Figure 3 with IMLM1. 

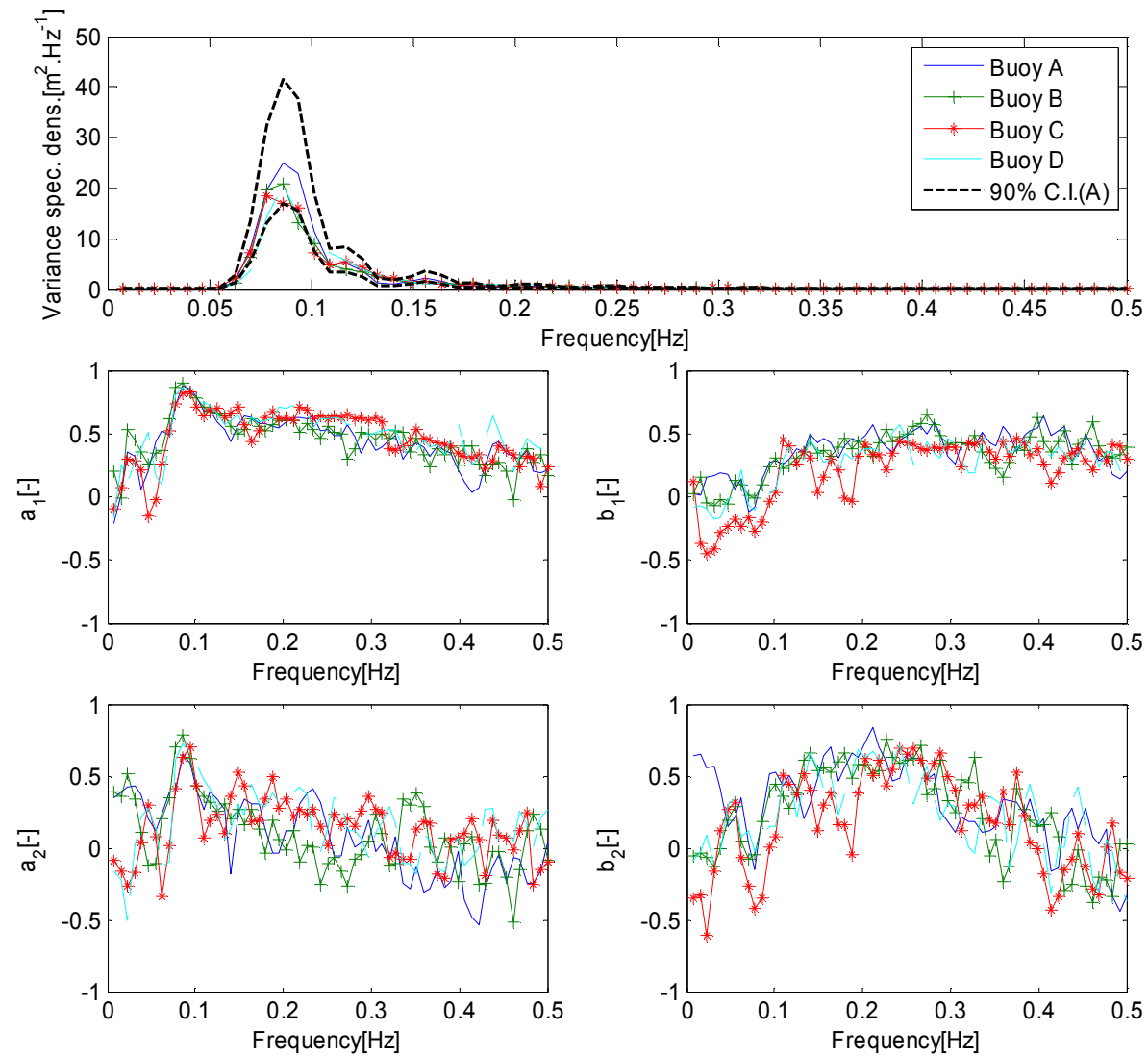

Figure 5: Spectral estimates from the four measurement buoys on the $5^{\text {th }}$ of February 2010, 9am: variance spectral density (top) with buoy-A-based 90\% confidence interval limits and directional Fourier coefficients $a_{1}, b_{1}$ (bottom left) and $a_{2}, b_{2}$ (bottom right). 

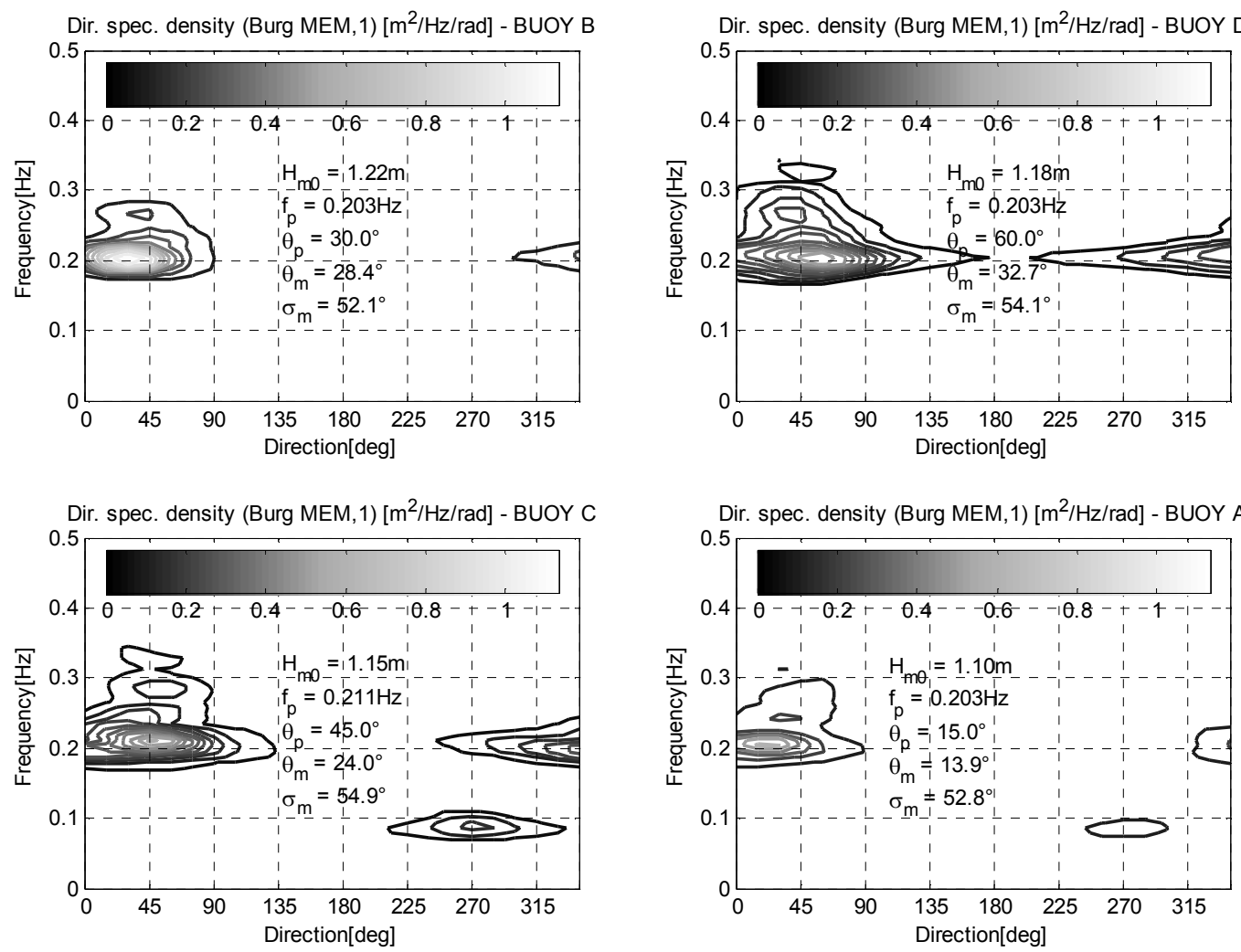

Figure 6: Directional spectra estimated with BMEM1 at the four measurement buoy locations on the $14^{\text {th }}$ of February 2010, 5am. 

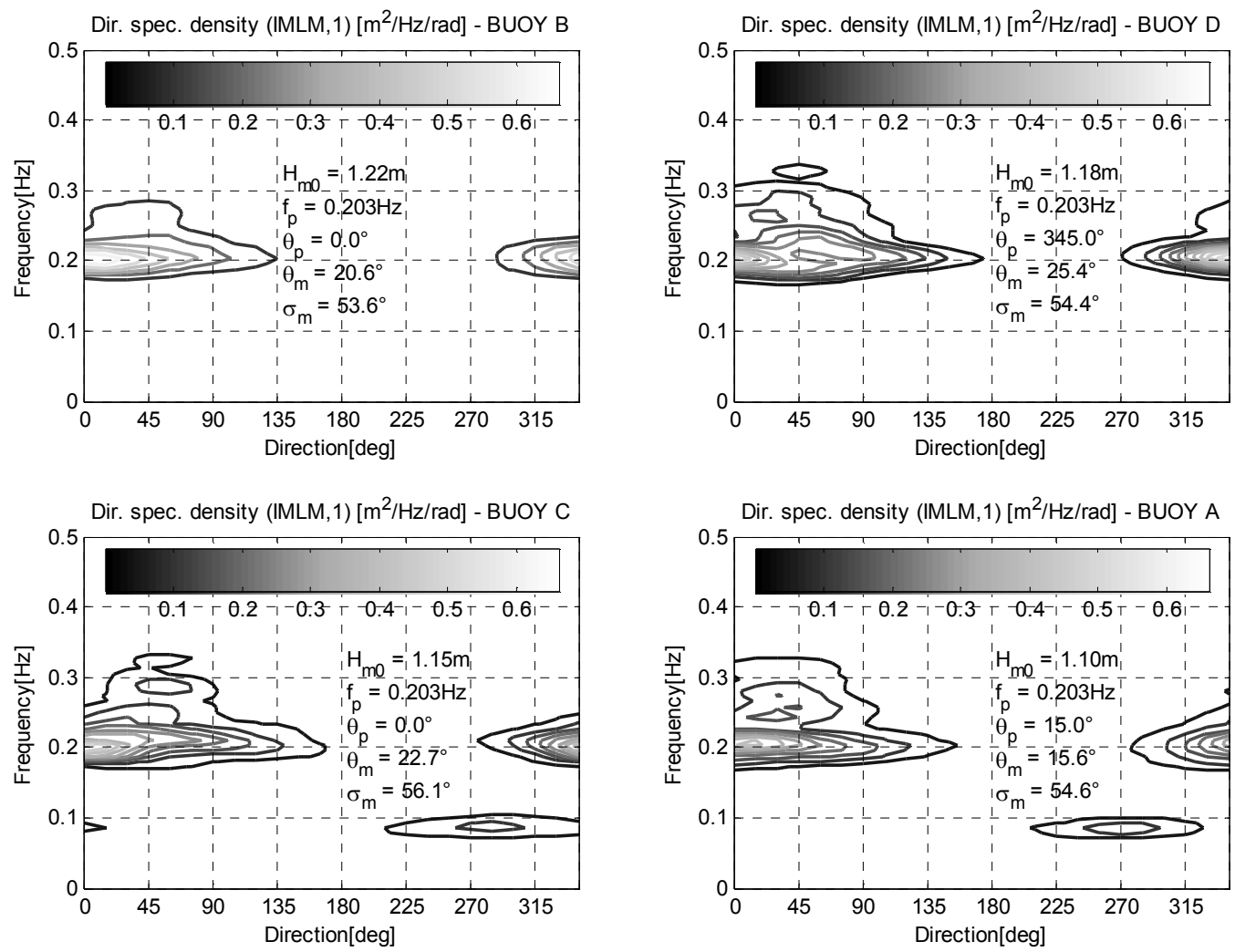

Figure 7: Idem Figure 6 with IMLM1. 

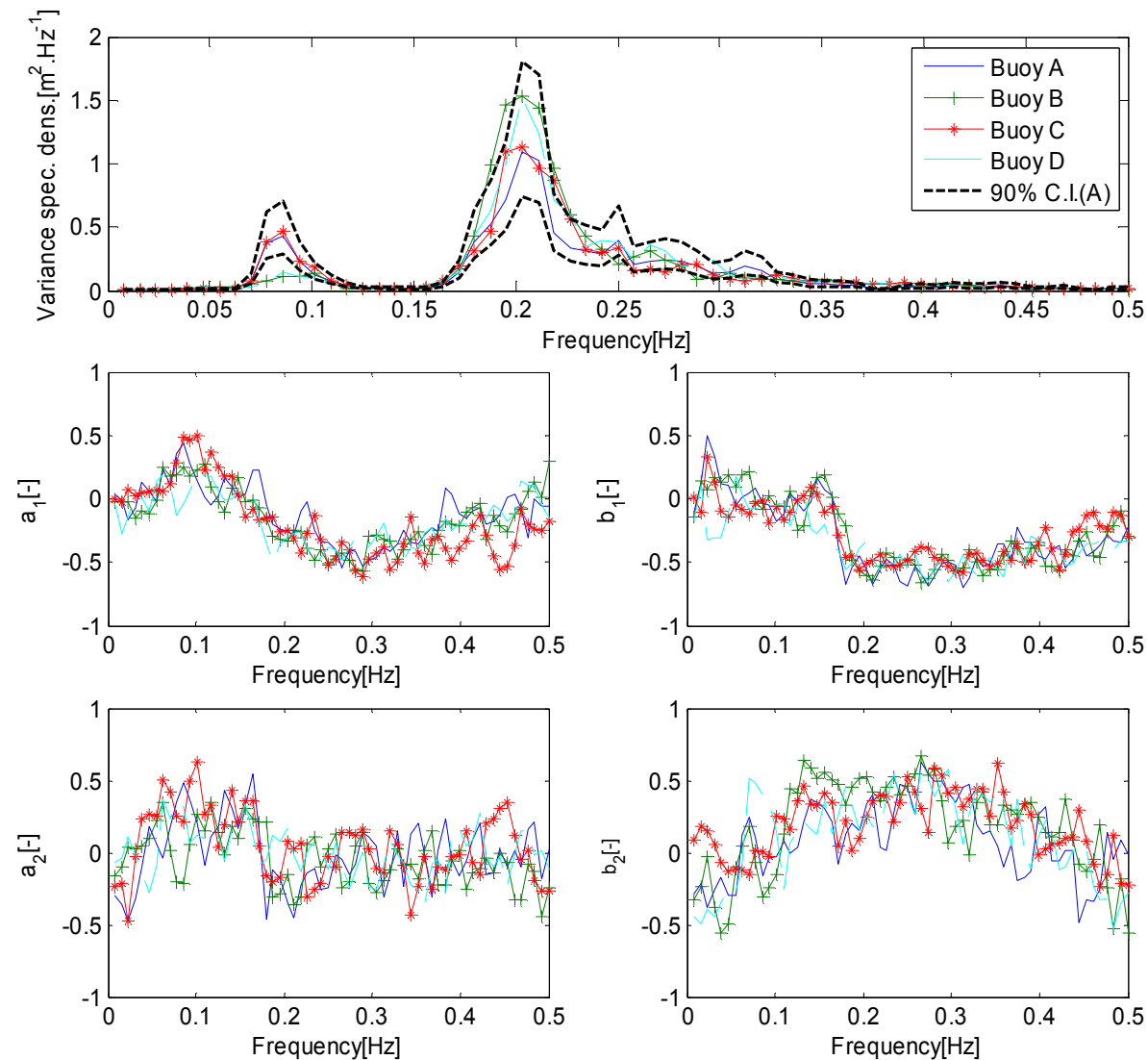

Figure 8: Idem Figure 5 on the $14^{\text {th }}$ of February 2010, $5 \mathrm{am}$. 

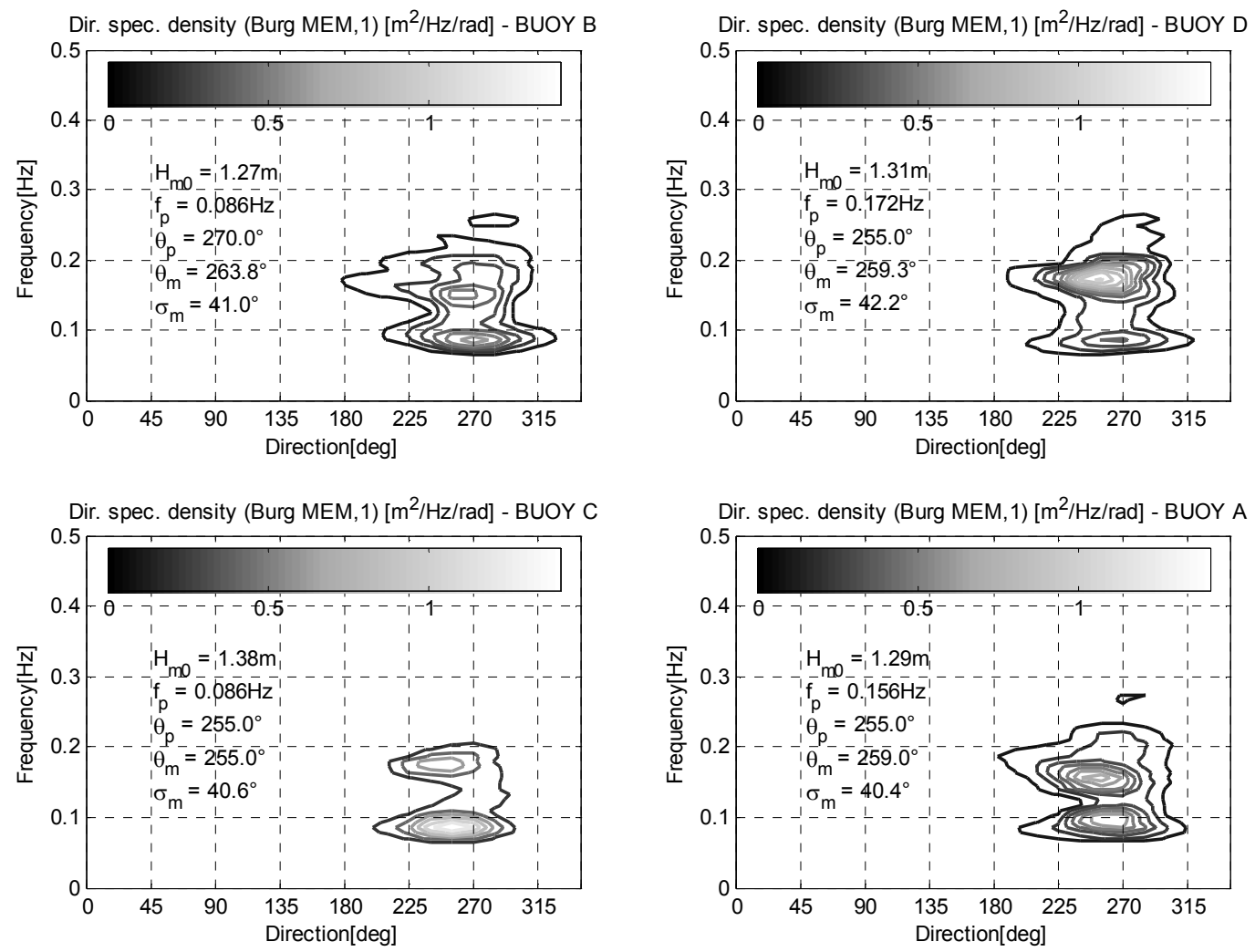

Figure 9: Directional spectra estimated with BMEM1 at the four measurement buoy locations on the $21^{\text {st }}$ of February 2010, 10am. 

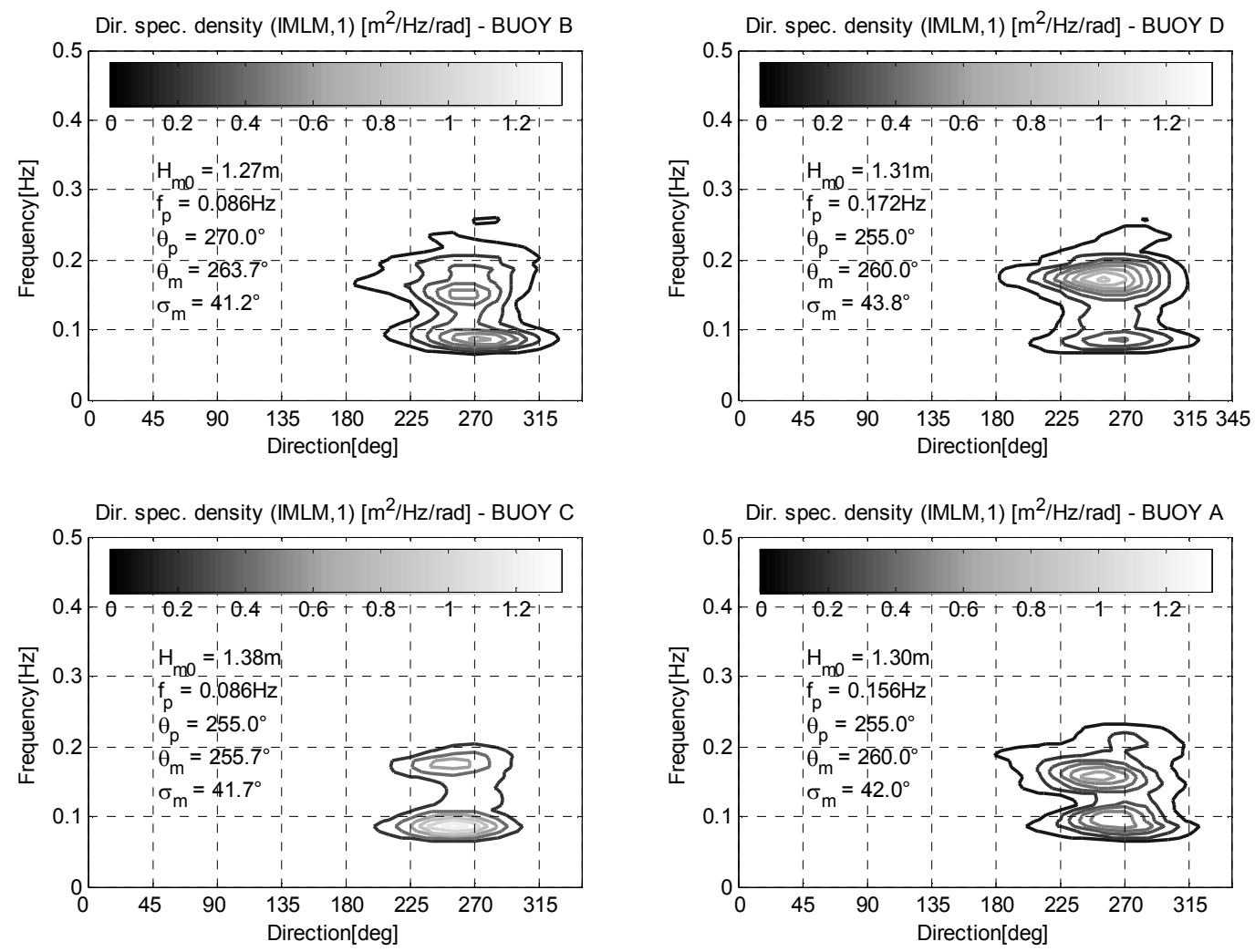

Figure 10: Idem Figure 9 with IMLM1. 

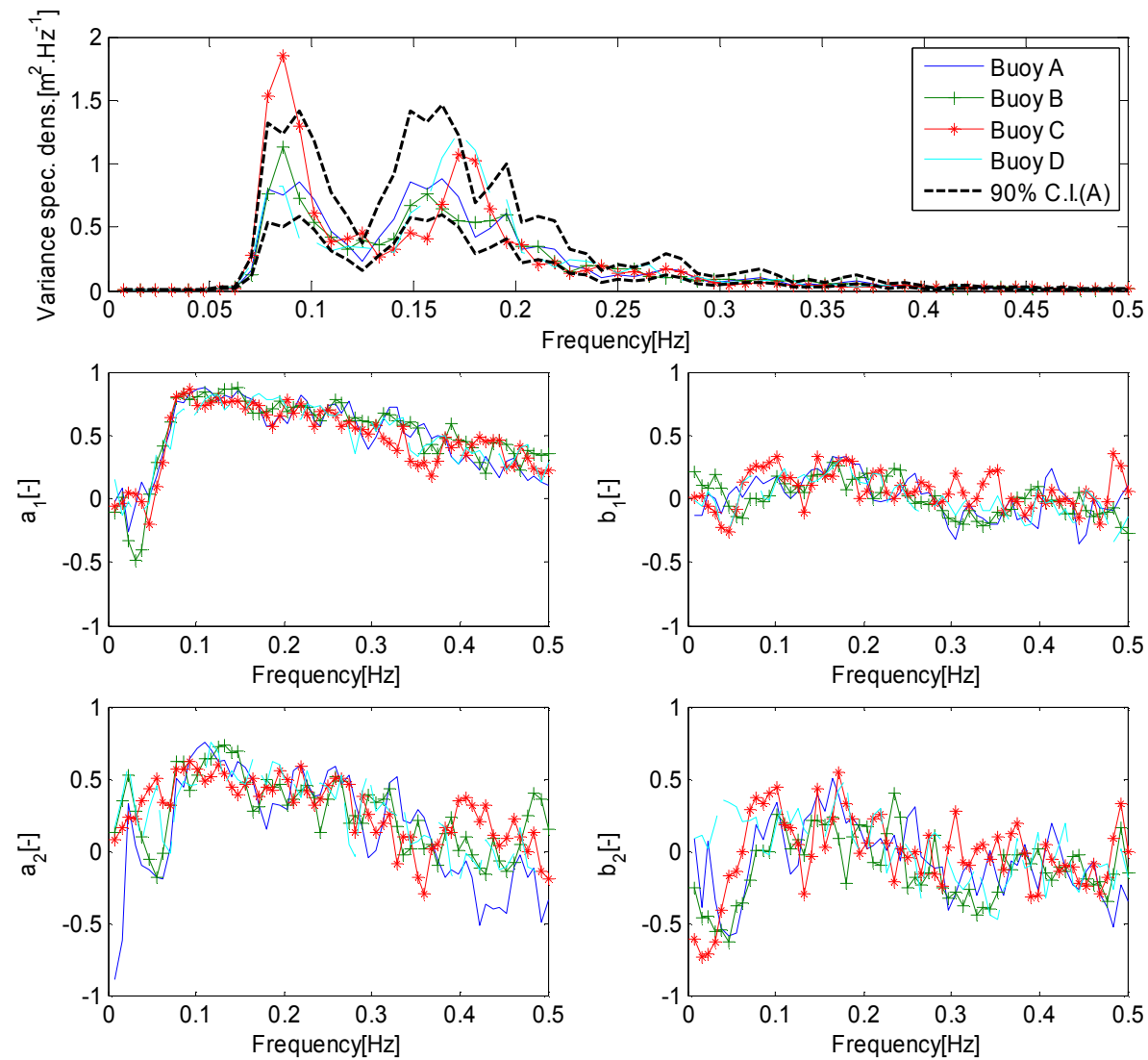

Figure 11: Idem Figure 5 on the $21^{\text {st }}$ of February 2010, 10am. 

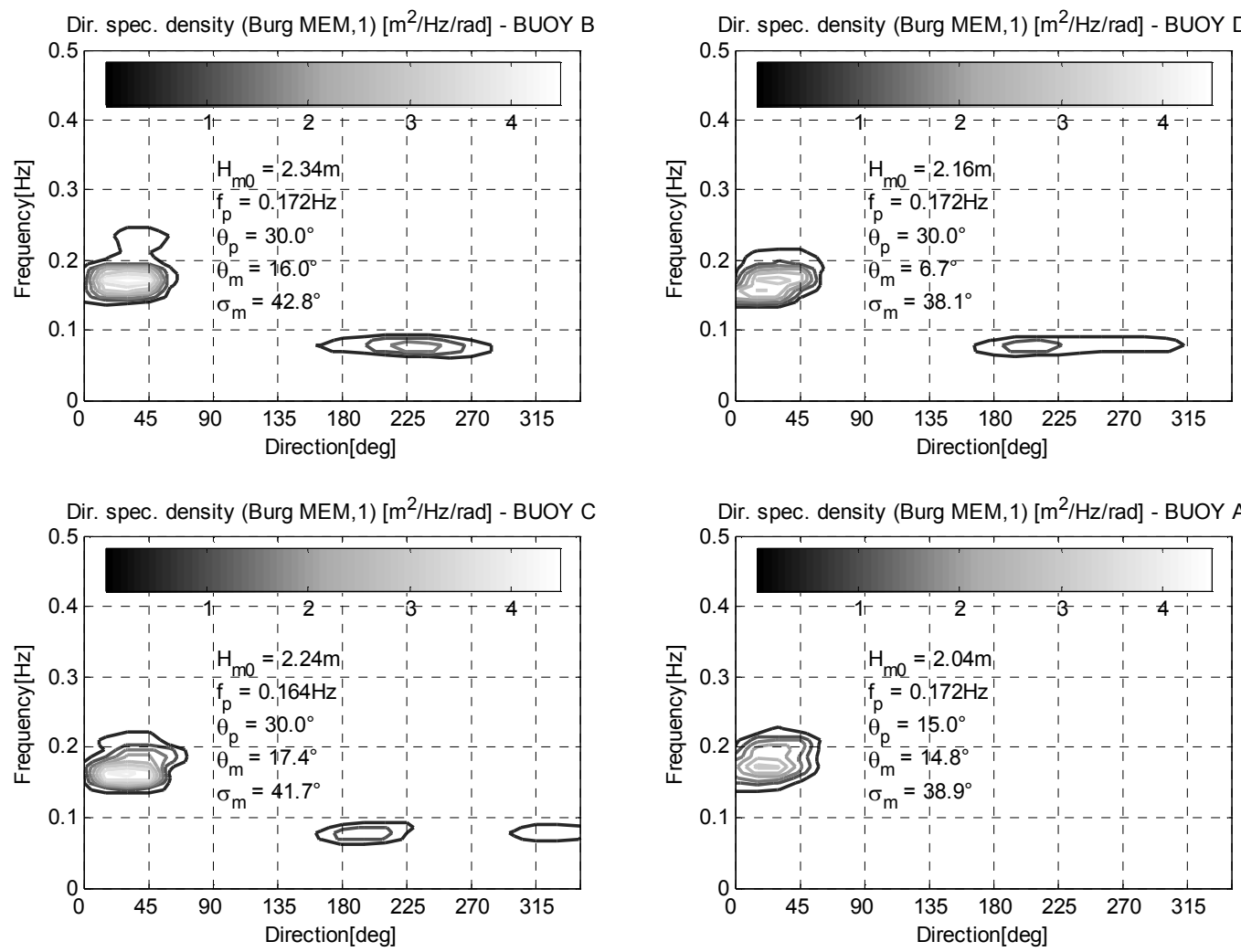

Figure 12: Directional spectra estimated with BMEM1 at the four measurement buoy locations on the $22^{\text {nd }}$ of February 2010, 7.30am. 

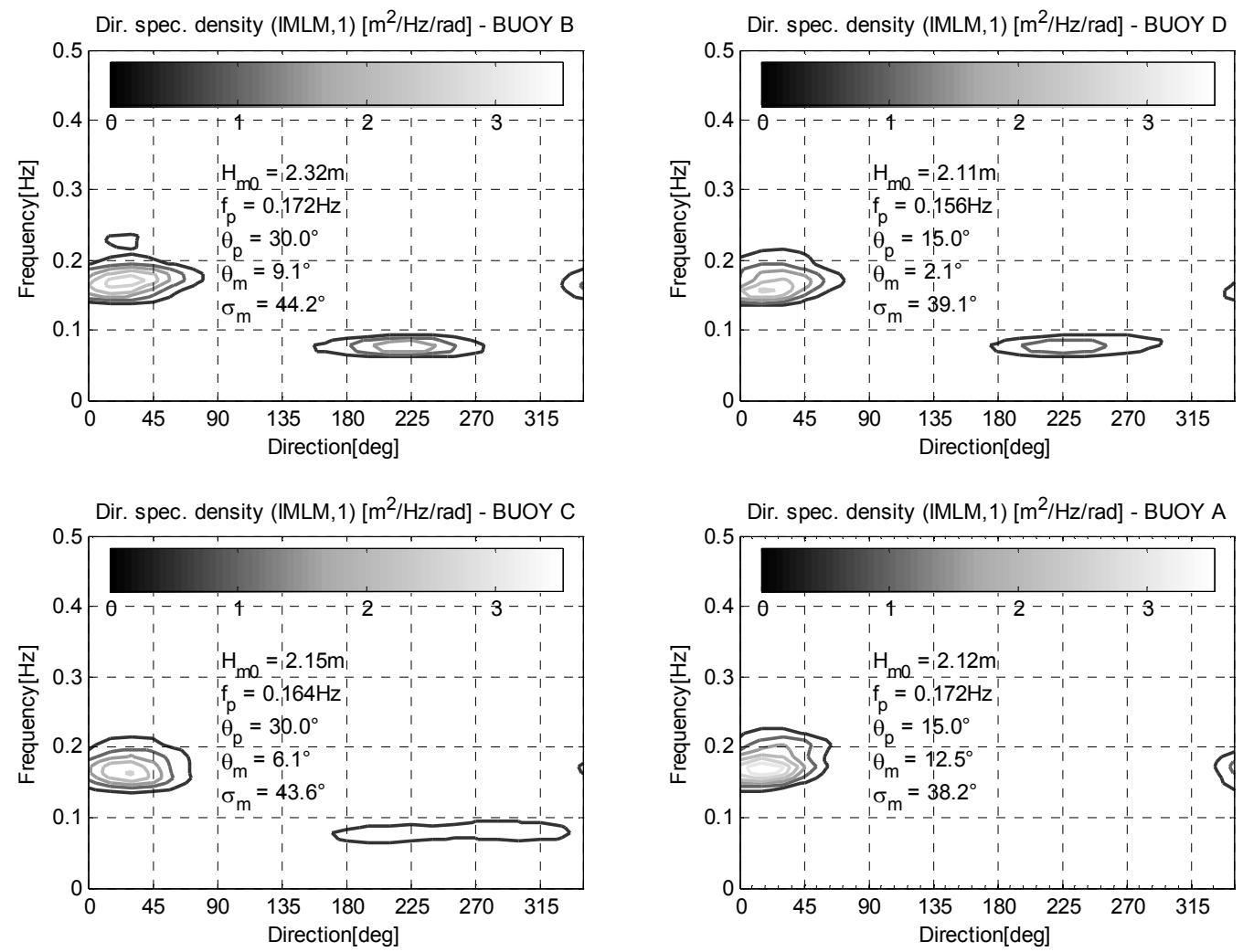

Figure 13: Idem Figure 12 with IMLM1. 

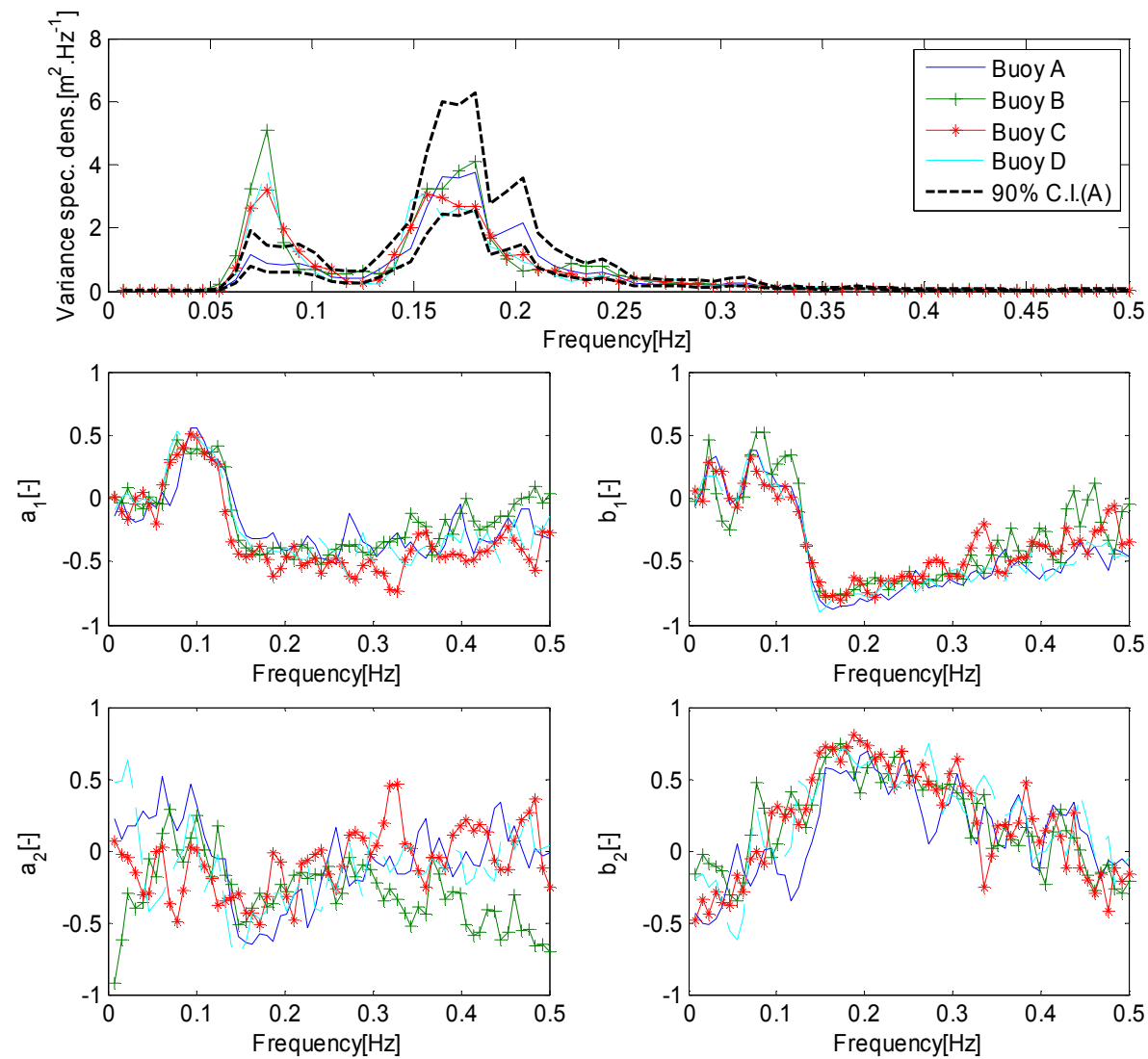

Figure 14: Idem Figure 5 on the $22^{\text {nd }}$ of February 2010, 7.30am. 

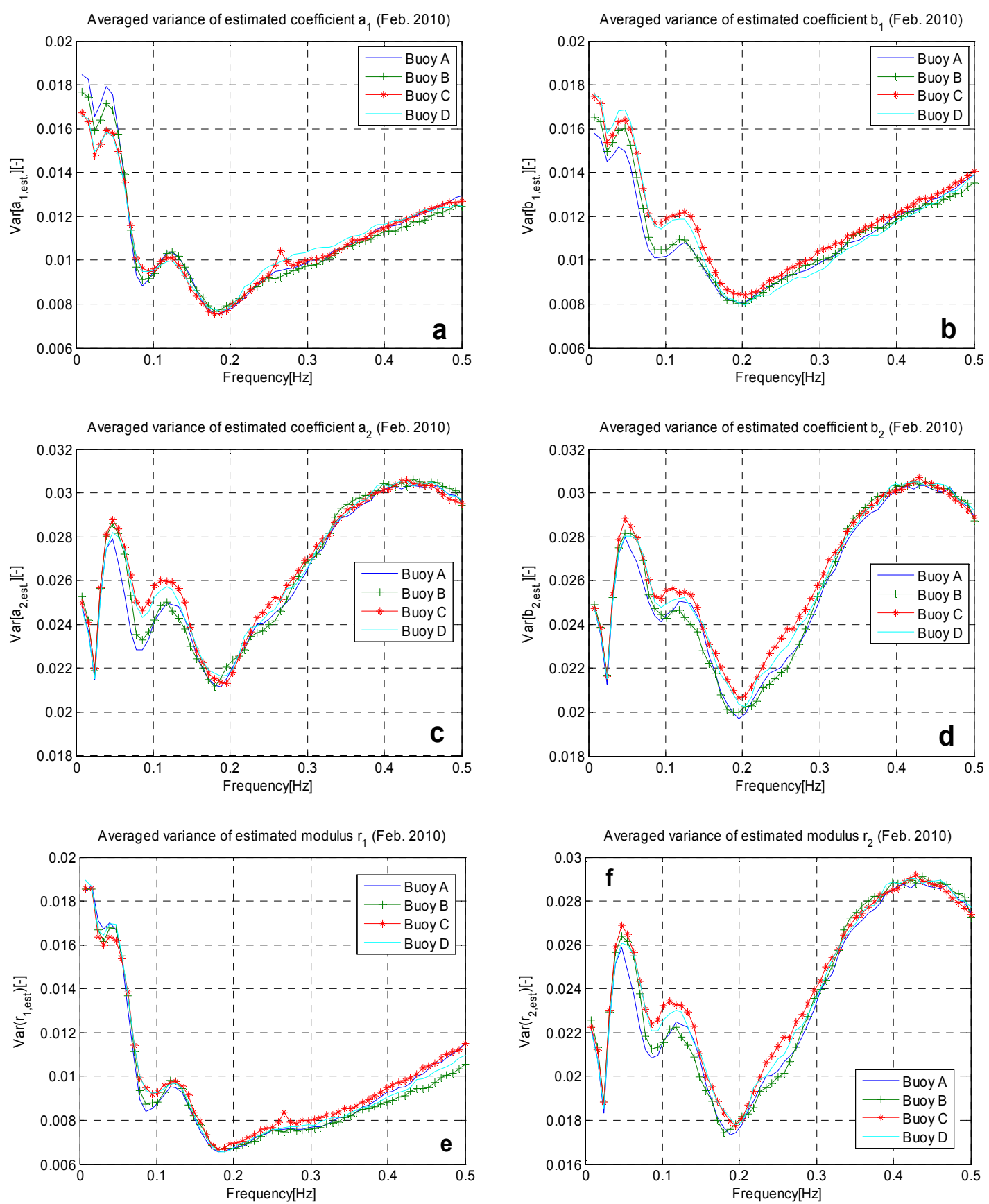

Figure 15: Average variance of estimated Fourier coefficients $a_{1}$ (a), $b_{1}$ (b), $a_{2}(\mathrm{c}), b_{2}$ (d) and modulus $r_{1}$ (d) and $r_{2}$ (e) (Eq. (27)) for the month of February 2010 over the buoy array. 


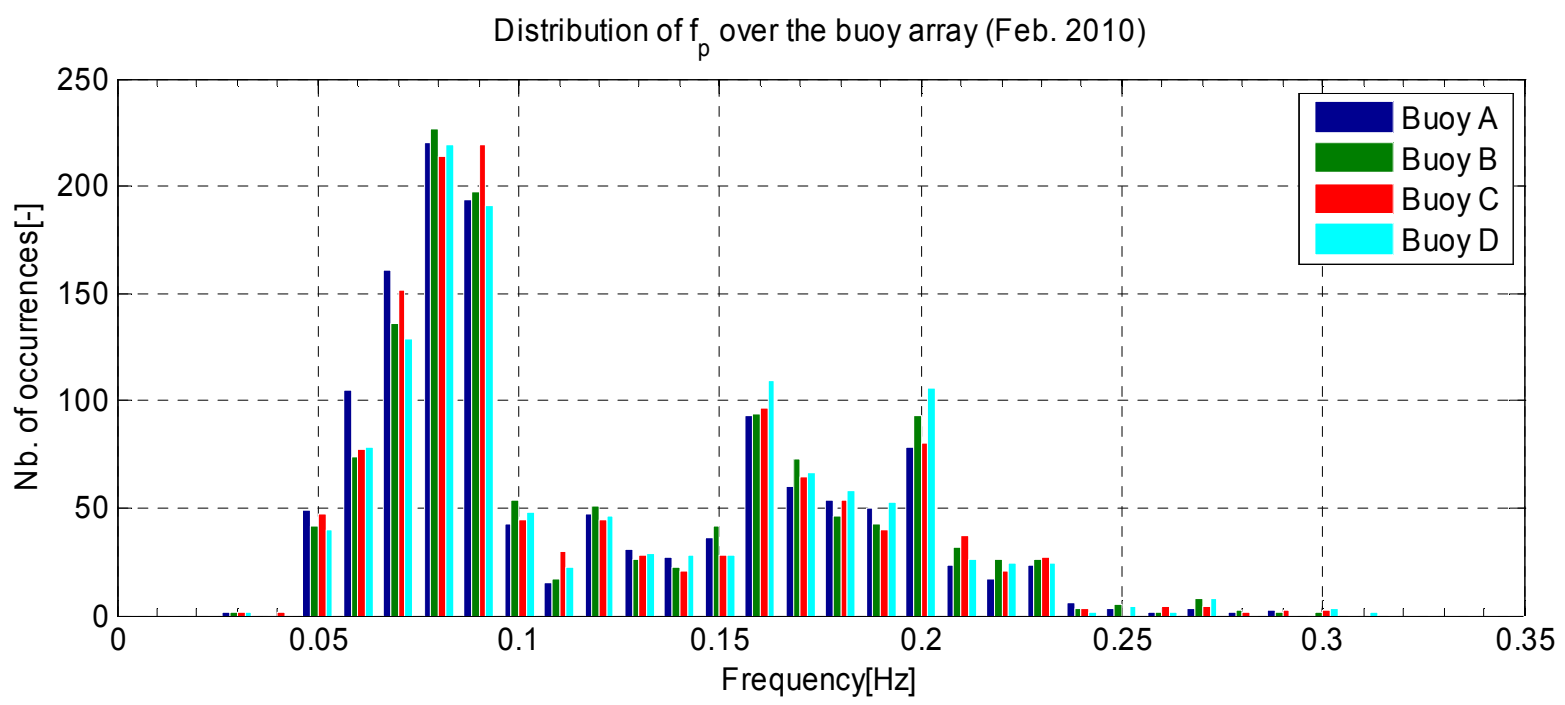

Figure 16: Distribution of overall peak frequency of the sea states estimated by the buoy array during February 2010 (0.01Hz-wide bins histogram). 

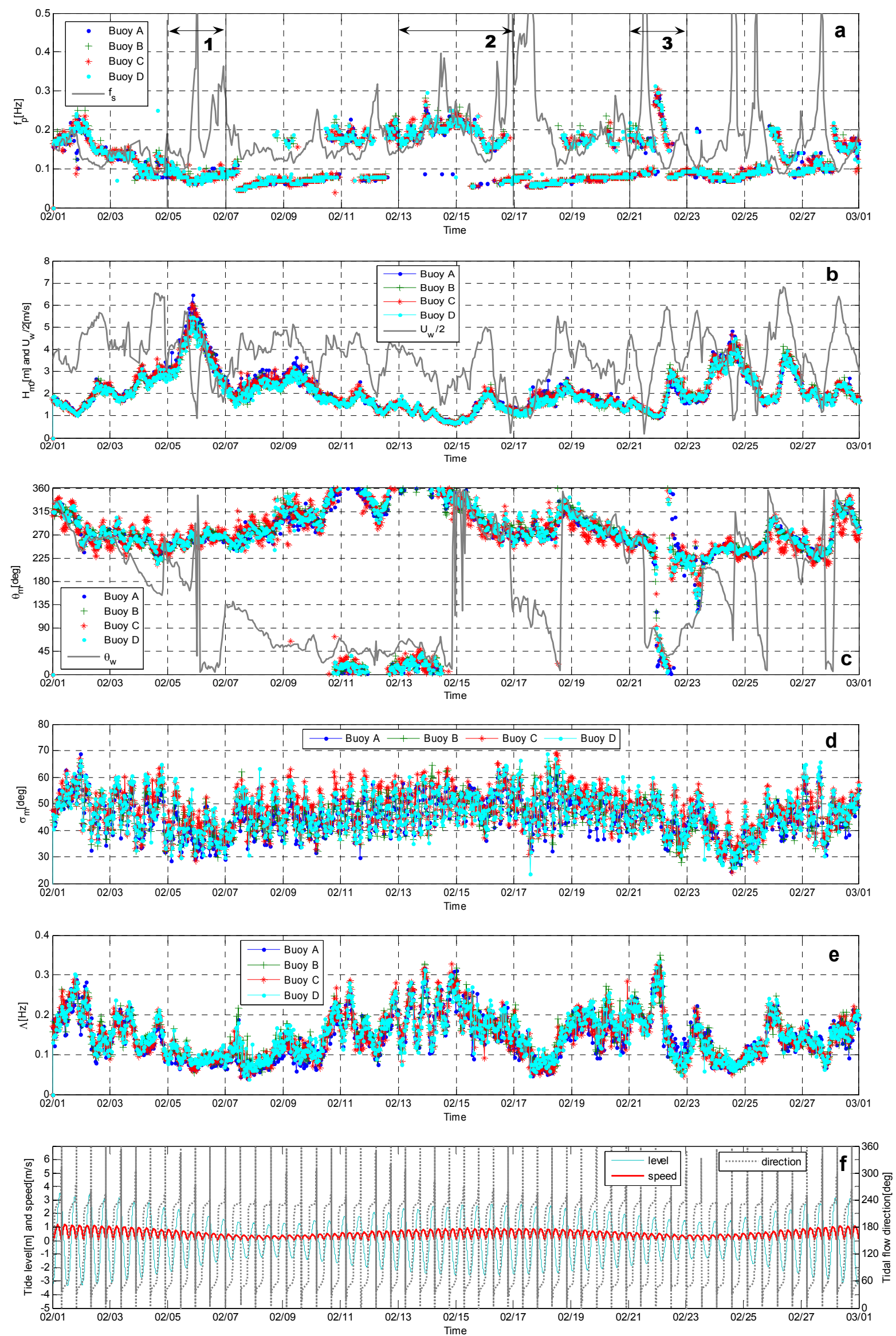

Figure 17: Overall parameters of sea states over the buoy array during February 2010 (BMEM1): peak period (a), significant wave height (b), mean direction (c), mean directional spreading (d) and spectral bandwidth (e); wind data (thin black line): separation frequency (a), wind speed divided by two (b) and wind direction (c); tide data (f). Windows 1, 2 and 3 (see Fig. 18) are highlighted in subplot (a). 

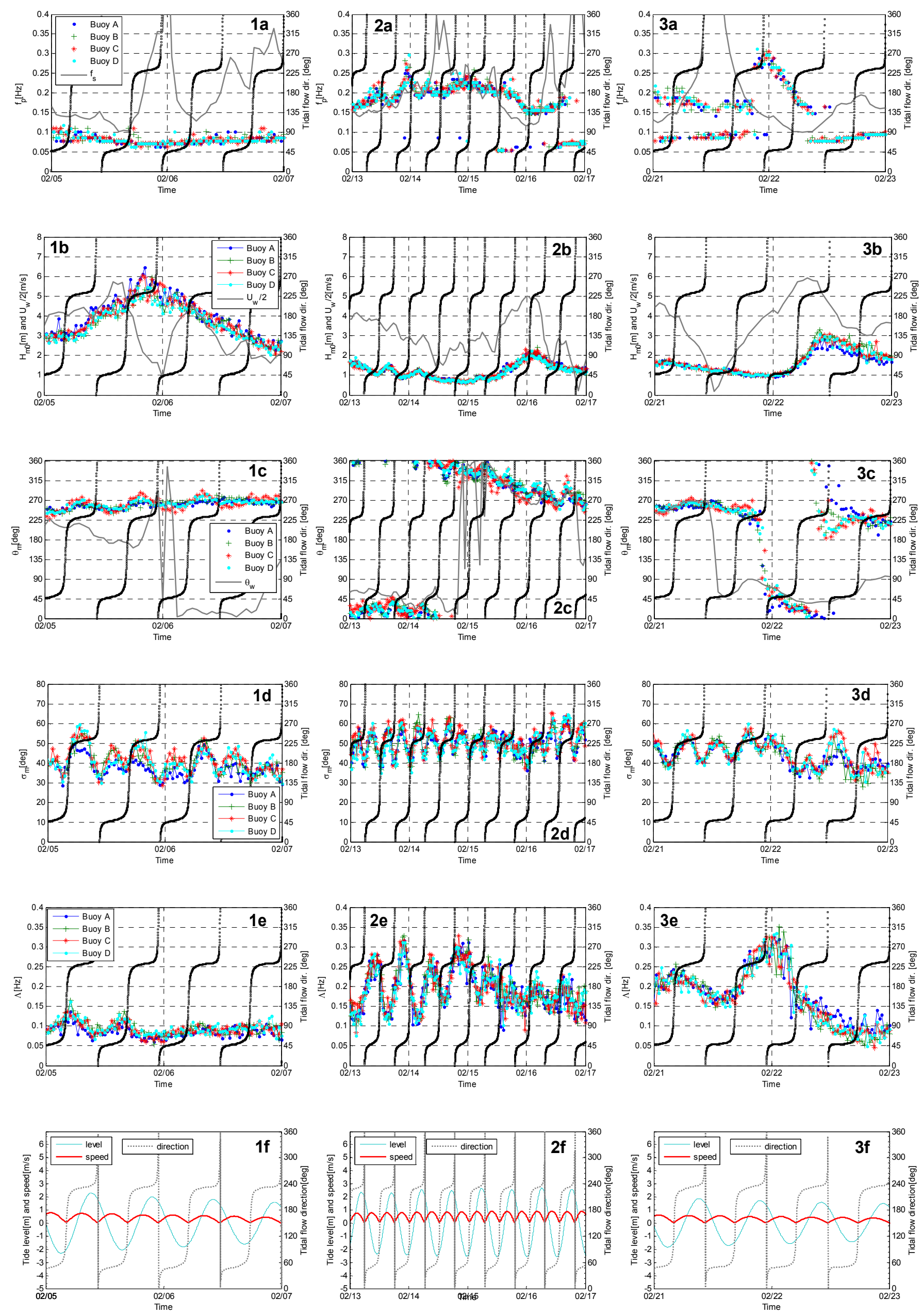

Figure 18: Idem Figure 17 for three particular time windows $\left(1: 5^{\text {th }}-7^{\text {th }}, 2: 13^{\text {th }}-17^{\text {th }}, 3: 21^{\text {st }}-23^{\text {rd }}\right.$ of February 2010$)$ 

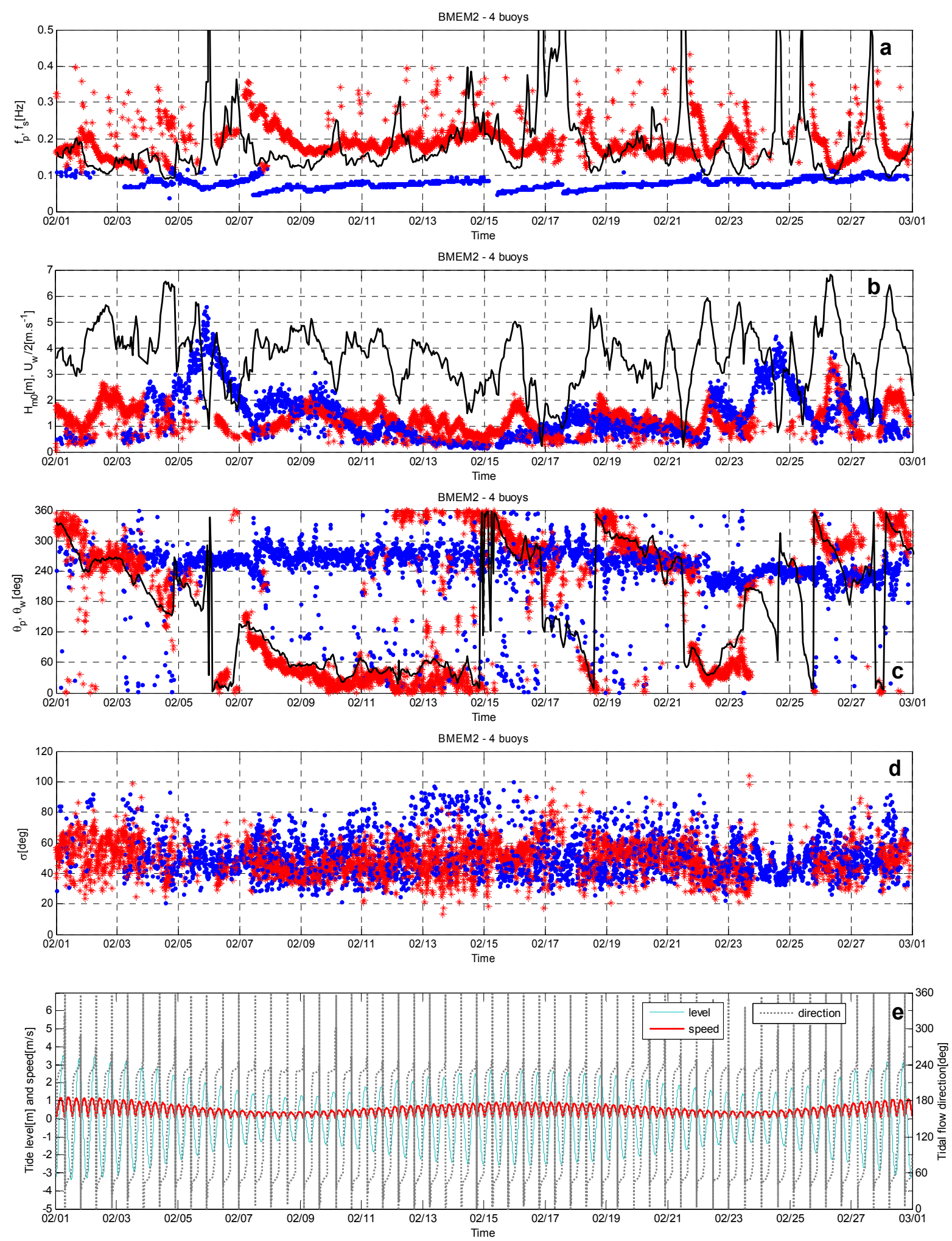

Figure 19: Characteristics of swell ('.') and wind-sea ('+') wave systems identified from the four measurement buoys during February 2010 (BMEM2): peak frequency (a), significant wave height (b), peak direction (c) and directional spreading (d); wind data: separation frequency (a), wind speed divided by two (b) and wind direction (c); tide data (e). 


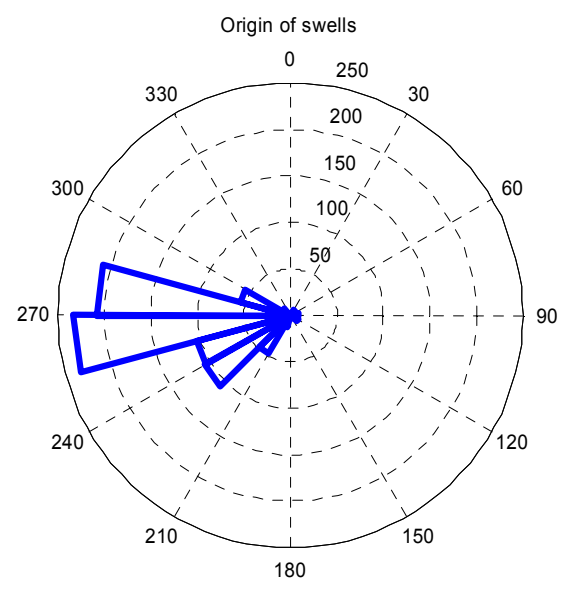

(a)

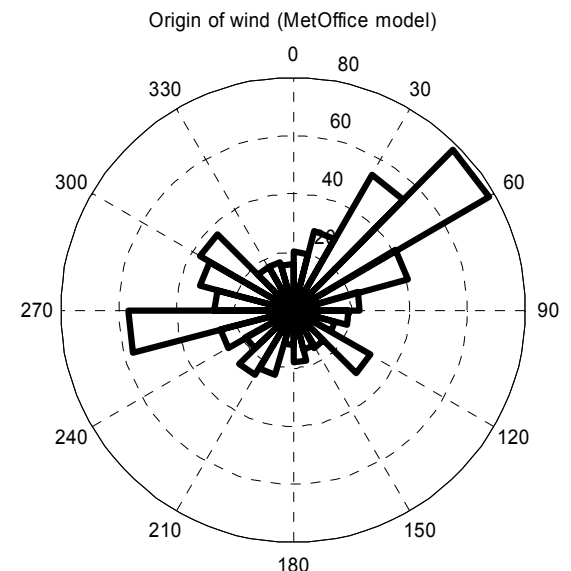

(c)

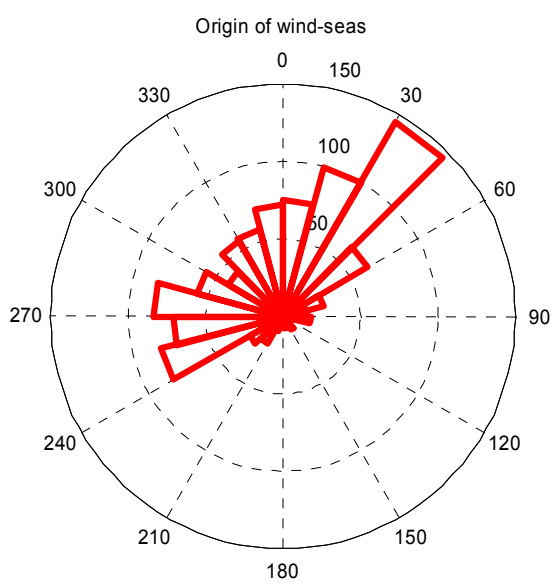

(b)

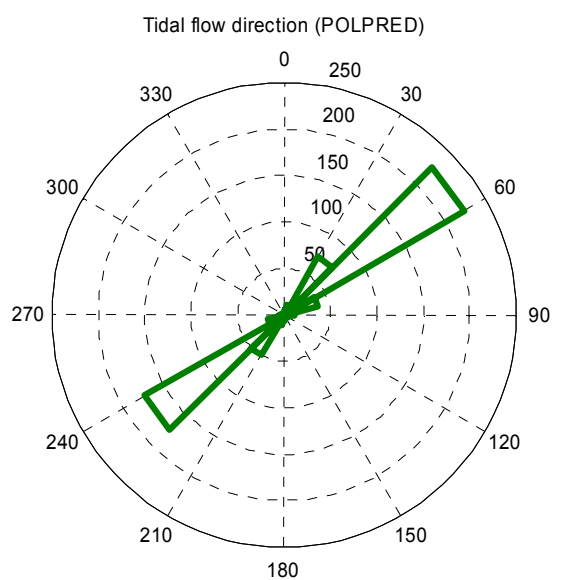

(d)

Figure 20: Polar histogram of swell (a) and wind-sea (b) systems (from buoy A tracking results), POLPRED model tidal data (c) and MetOffice wind model (d) in February 2010. 

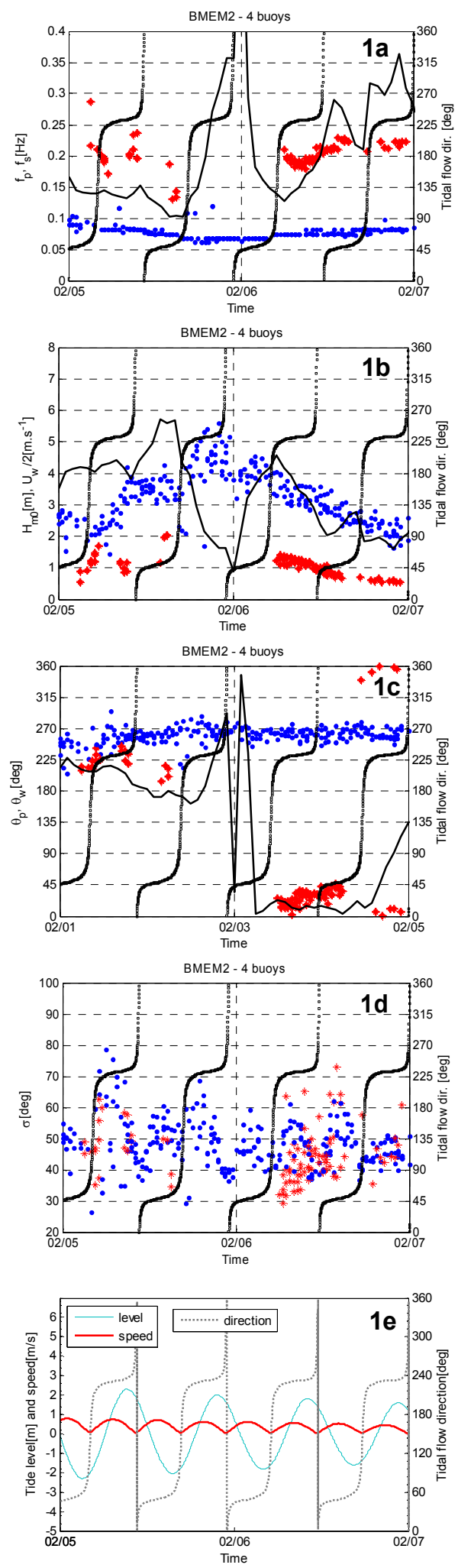
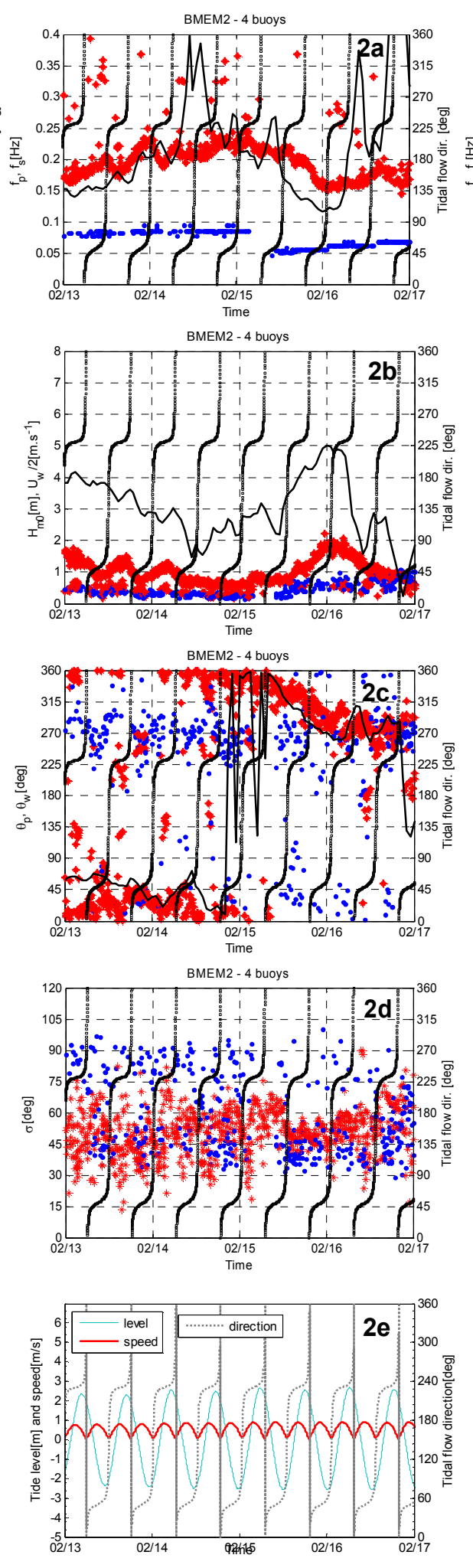
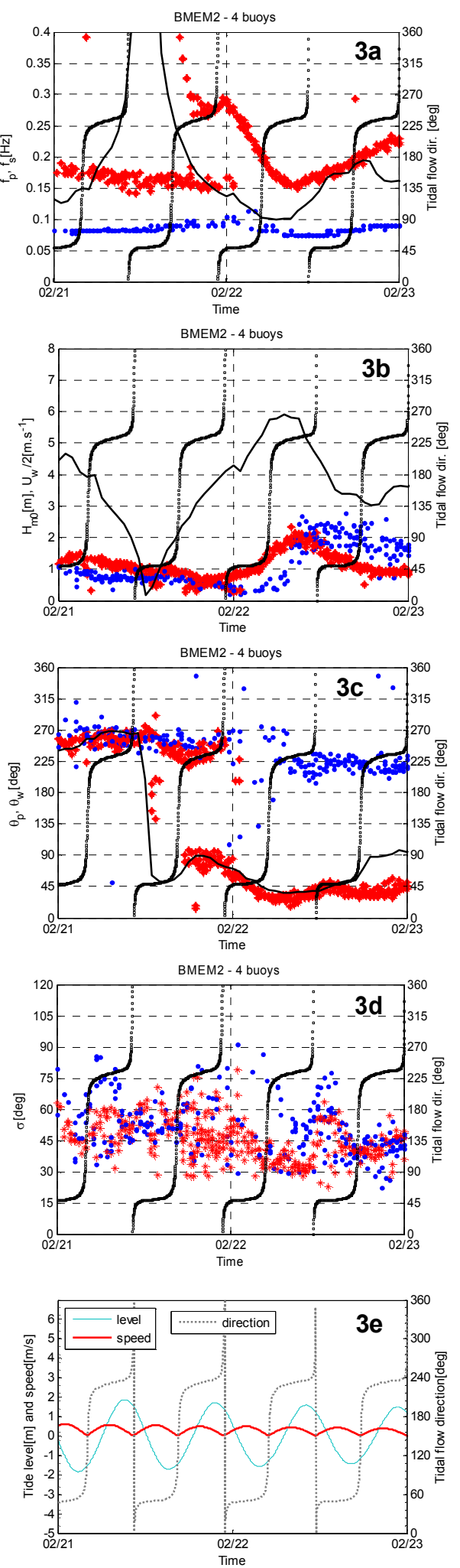

Figure 21: Idem Figure 19 for three particular time windows (see Fig. 18); tidal flow direction (from) is plotted with square markers in subfigures a to $d$. 